\title{
5 Anhang
}





\subsection{Anhang I}

Erstbelege für Papiermühlen im Reich bis 1500 (außer Reichsitalien) 

Die folgende Tabelle stellt die Erstbelege für Papiermühlen im Reich vor 1500 unter Auslassung Reichsitaliens zusammen. Sie basiert auf der Forschungsliteratur zu den einzelnen Papiermühlenstandorten. Nicht alle Angaben sind jedoch durch Quellenbelege gesichert. Dies gilt vor allem für die Ausführungen von Friedrich von Hößle, Hans Heinrich Bockwitz und Viktor Thiel, sodass diese Daten bis zu einer Überprüfung an Archivmaterial unsicher bleiben. Die Erstbelege sind zudem von unterschiedlicher Qualität. Einige weisen dezidiert eine Papiermühle nach, andere bezeugen lediglich die Anwesenheit eines Papiermachers oder - wie im Fall von Lüneburg - den Import von Lumpen, weitere stützen sich lediglich auf Wasserzeichen.

Mit den Papiermühlenzahlen, die Alfred Schulte und Viktor Thiel in ihren Werken angeben, sind die hier ermittelten Zahlen in zweierlei Hinsicht nicht vergleichbar. Zum einen werden in der nachfolgenden Tabelle die Erstbelege für einen Papiermühlenstandort angeführt, nicht jedoch weitere Papiermühlen, die nach diesem Datum entstanden. Die Anzahl der Papiermühlenstandorte im untersuchten Raum ist folglich kleiner als die Anzahl der Papiermühlen. Zum anderen erhoben Schulte und Thiel ihre Daten für das Dritten Reich und decken somit einen anderen Raum ab. ${ }^{3003}$ Viktor Thiel zählte zehn bis zwölf Papiermühlen, die vor 1450 entstanden, und insgesamt 40 Betriebe, die vor 1500 die Produktion aufnahmen. ${ }^{3004}$ Diese Schätzungen sind nicht unumstritten: Die Zahl der von Thiel angegebenen 25 Papiermühlen im alemannischen Raum erscheint plausibel, insgesamt schätzt Alfred Schulte die Anzahl der Papiermühlen auf deutschem Boden bis 1500 jedoch auf 60 Papiermühlen. ${ }^{3005}$ Günter Bayerl legt seiner Untersuchung der Papiermühlen vom Ende des 14. bis zum Beginn des 20. Jahrhunderts das Reich in den Grenzen des späten 18. Jahrhunderts zugrunde, sodass ein Großteil der linksrheinischen Gebiete aus seiner Betrachtung herausfällt. $^{3006}$ Bei der Zählung der Papiermühlenstandorte ist zudem immer zu beachten, dass womöglich bereits vor 1500 einige Papiermühlen die Produktion einstellten, wie es für die erste Nürnberger Papiermühle nachweisbar ist. ${ }^{3007}$ Wenn also bis 1500 an insgesamt 74 Standorten im Reich Papiermühlen eingerichtet wurden, dann folgt daraus nicht zwangläufig, dass um 1500 noch an 74 Standorten Papier hergestellt wurde.

3003 Vgl. Alfred Schulte 1934, 9-11; V. Thiel 1941, 31-43.

3004 Vgl. V. Thiel 1941, 37.

3005 Vgl. Alfred Schulte 1934, 11. In seiner Nachfolge Schlieder 1966, 114.

3006 Vgl. Bayerl 1987, 593-597.

3007 Vgl. Sporhan-Krempel 1990b, 191 f.; Stromer 1990a, 34. 


\begin{tabular}{|c|c|c|c|}
\hline & Jahr & Ort & Literatur/Belege \\
\hline 1. & 1375 & Schopfheimi & Kälin 1974, 83-87. \\
\hline 2. & 1390 & Nürnberg & Vgl. Anm. 2. \\
\hline 3. & $1392 / 1402$ & Ravensburg & Vgl. Kapitel 3.2.1.2. \\
\hline 4. & 1398 & Chemnitzii & F. H. Meyer 1888, 286. \\
\hline 5. & 1409 & Rothenburg ob der Tauber & Stromer/Sporhan-Krempel 1963, 71. \\
\hline 6. & $1418 / 1469$ & Verdun & Zaar-Görgens 2004, 30, 263. \\
\hline 7. & 1420 & Schönkamp bei Lübeck & Schlieder 1966, 95-98. \\
\hline 8. & 1420 & Altenbeckern bei Liegnitziii & Bockwitz 1935, 94. \\
\hline 9. & 1426 & Allemogne bei Genf & P. Tschudin 1991, 21. \\
\hline 10. & 1428 & Gennep & Alfred Schulte 1932, 44-47. \\
\hline 11. & 1428 & Hönhagen bei Nusse & Schlieder 1966, $96 \mathrm{f}$. \\
\hline 12. & $1431 / 1476$ & Lüneburg & Bockwitz 1935, 95; Schlieder 1966, 99. \\
\hline 13. & 1432 & Belfaux & Gerardy 1980a, 51 f. \\
\hline 14. & 1433 & Schwabach & Bockwitz 1935, 95. \\
\hline 15. & 1437 & Huy & Arnould 1976, $289 \mathrm{f}$. \\
\hline 16. & 1439 & Linkebeek & Van Wegens 2015, $78 \mathrm{f}$ \\
\hline 17. & 1440 & Basel & Vgl. Kapitel 3.2.1.1. \\
\hline 18. & $1443 / 1447$ & Bautzen & W. Weiss 1990, 63. \\
\hline 19. & $1444 / 1464$ & Épinal & $\begin{array}{l}\text { Janot 1952, 321-323; Zaar-Görgens 2004, } 43 \text {, } \\
261 .\end{array}$ \\
\hline 20. & 1445 & Straßburgiv & Vgl. Kapitel 3.2.1.15. \\
\hline 21. & 1445 & La Glâne bei Ecuvillens & Gerardy 1980a, 53. \\
\hline 22. & $1445 / 1494$ & Schaerbeek & Van Wegens 2015, 81, 88 \\
\hline
\end{tabular}

i Hans Kälin, der in den Basler Rechnungsbüchern Schopfheim als Bezugsort von Papier ausmachen konnte, bezweifelt selbst jedoch, dass es sich dabei um einen Papiermühlenstandort handelte. Im Gegensatz dazu hält Franz Irsigler diesen Befund für stichhaltig, vgl. Irsigler 1999, 258-260.

ii Für Chemnitz existiert nur ein Privileg Wilhelms I. von Meißen, das den Bau einer Papiermühle gestattet. Ob diese Mühle gebaut wurde, ist unsicher, vgl. F. H. Meyer 1888, 286.

iii Wolfgang Schlieder zweifelt die Existenz einer Papiermühle bei Altenbeckern an, vgl. Schlieder 1966, 99.

iv Lange Zeit wurde aufgrund von Wasserzeichen eine Papiermühlengründung in Straßburg im Jahr 1408 angenommen, vgl. u. a. Bockwitz 1935, 94. Diese Vermutung konnte nicht bestätigt werden, vgl. Kapitel 3.2.1.15. 


\begin{tabular}{|c|c|c|c|}
\hline & Jahr & Ort & Literatur/Belege \\
\hline 23. & 1446 & Metz & $\begin{array}{l}\text { Zaar-Görgens 1995, 177; Zaar-Görgens 2004, } \\
33 \text { f. }\end{array}$ \\
\hline 24. & 1450 & Sint-Lambrechts-Woluwe & Van Wegens 2015, 81, 88 \\
\hline 25. & 1451 & Coburg & Stromer 1990a, 34; Stromer 1990c, 166. \\
\hline 26. & $\begin{array}{l}\text { zw. } 1460 \text { u. } \\
1486\end{array}$ & Vallières & Zaar-Görgens 2004, 39, 263. \\
\hline 27. & 1461 & Ettlingen & Vgl. Kapitel 3.2.1.11. \\
\hline 28. & 1462 & Diegem & Van Wegens 2015, $81 \mathrm{f}$. \\
\hline 29. & $1462 / 63$ & Frouard & Zaar-Görgens 2004, 60, 261. \\
\hline 30. & 1463 & Vieux-Thann & Vgl. Kapitel 3.2.1.16. \\
\hline 31. & 1464 & St. Dié & Zaar-Görgens 2004, 263. \\
\hline 32. & um 1465 & Söflingen & Vgl. Kapitel 3.2.1.6. \\
\hline 33. & 1466 & Bern & Vgl. Kapitel 3.2.1.7. \\
\hline 34. & 1466 & Froideconche & Hößle 1925, 231. \\
\hline 35. & 1468 & Augsburg & Vgl. Kapitel 3.2.1.3. \\
\hline 36. & 1469 & St. Pölten & V. Thiel 1940, 26, 28. \\
\hline 37. & 1469 & Arches & Briquet 1968, 169 f.; Zaar-Görgens 2004, 260. \\
\hline 38. & 1469 & Schniegling bei Nürnberg & Stromer 1990c, 169. \\
\hline 39. & $1470 / 1471$ & Archettes & Janot 1952, 133 f.; Zaar-Görgens 2004, 260. \\
\hline 40. & $1470 / 1489$ & Reutlingen & Vgl. Kapitel 3.2.1.10. \\
\hline 41. & 1471 & Zürich & Vgl. Kapitel 3.2.1.8. \\
\hline 42. & $1472 / 1497$ & Lörrach & Vgl. Kapitel 3.2.1.12. \\
\hline 43. & 1474 & Marly ${ }^{v}$ & Gerardy 1980a, 55 f. \\
\hline 44. & 1475 & Kraainem & Van Wegens 2015, 82, 89 \\
\hline 45. & $1476 / 1478$ & Braine l'Alleud & Van Wegens 2015, 82, 89 \\
\hline 46. & 1477 & Bains-les-Bains & Janot $1952,151 \mathrm{f}$. \\
\hline 47. & 1477 & Kempten & Vgl. Kapitel 3.2.1.4. \\
\hline 48. & 1477 & Serrières bei Neuenburg & P. Tschudin 1991, 33. \\
\hline 49. & 1477 & Urach & Vgl. Kapitel 3.2.1.9. \\
\hline
\end{tabular}

v Das Jahr 1411 als Gründungsdatum der Papiermühle in Marly bei Freiburg im Üchtland kann seit den Forschungen von Theodor Gerardy als widerlegt gelten. Vgl. hierzu Bockwitz 1935, 94; P. Tschudin 1991, 19. 


\begin{tabular}{|c|c|c|c|}
\hline & Jahr & Ort & Literatur/Belege \\
\hline 50. & $1478 / 1482$ & Memmingen & Vgl. Kapitel 3.2.1.5. \\
\hline 51. & 1479 & Zaventem & Van Wegens 2015, 82, 89. \\
\hline 52. & 1480 & Dieulouard & Zaar-Görgens 2004, 62, 261. \\
\hline 53. & 1480 & St. Léonard & Zaar-Görgens 2004, 263. \\
\hline 54. & 1483 & Offenburg & Vgl. Kapitel 3.2.1.14. \\
\hline 55. & 1485 & Dresden & $\begin{array}{l}\text { Bockwitz 1935, 95; V. Thiel 1941, 42; Doss } 1993 \text {, } \\
21 .\end{array}$ \\
\hline 56. & 1488 & Champigneulles & Choux 1990, 54. \\
\hline 57. & 1488 & Gengenbach & Vgl. Kapitel 3.2.1.13. \\
\hline 58. & 1489 & Landshut & Hößle 1924, 331; Mitterwieser 1933, 16. \\
\hline 59. & 1489 & Kaiserslautern & Geldner 1962/64, 45. \\
\hline 60. & $\begin{array}{l}\text { zw. } 1489 \text { u. } \\
1505\end{array}$ & Siegburg & Gansen 1941, 21. \\
\hline 61. & 1490 & Breslau & Bockwitz 1935, 96; V. Thiel 1941, 41. \\
\hline 62. & 1490 & Au bei München & Hößle 1924, 234 f.; Mitterwieser 1940, 25. \\
\hline 63. & 1491 & Świdnica (dt. Schweidnitz) & Bockwitz 1935, 96; V. Thiel 1941, 41. \\
\hline 64. & 1492 & Leipzig & Bockwitz 1935, 96. \\
\hline 65. & 1492 & Docelles & Janot 1952, 270 f.; Zaar-Görgens 2004, 261. \\
\hline 66. & 1493 & Löwen & Arnould 1976, $279 \mathrm{f}$. \\
\hline 67. & 1493 & Rambervillers & Janot 1952,442 \\
\hline 68. & 1496 & Nysa (dt. Neisse) & Bockwitz 1935, 96; V. Thiel 1941, 41. \\
\hline 69. & 1497 & Racibórz (dt. Ratibor) & Bockwitz 1935, 96; V. Thiel 1941, 41. \\
\hline 70. & 1497 & Cernay (dt. Sennheim) & Vgl. Kapitel 3.2.1.16. \\
\hline 71. & 1497 & Erfurt & W. Weiss 1990, 63. \\
\hline 72. & 1498 & Wiener Neustadt & V. Thiel 1940, 26, 28. \\
\hline 73. & 1499 & Königssaal bei Prag & Bockwitz 1935, 96; V. Thiel 1941, 41. \\
\hline 74. & 1499 & Trutnov (dt. Trautenau) & Bockwitz 1935, 96; V. Thiel 1941, 41. \\
\hline
\end{tabular}




\subsection{Anhang II}

Produktionsleistung und Lumpenbedarf einer Papiermühle mit einer Bütte bei 300 Arbeitstagen im Jahr nach verschiedenen Autoren 



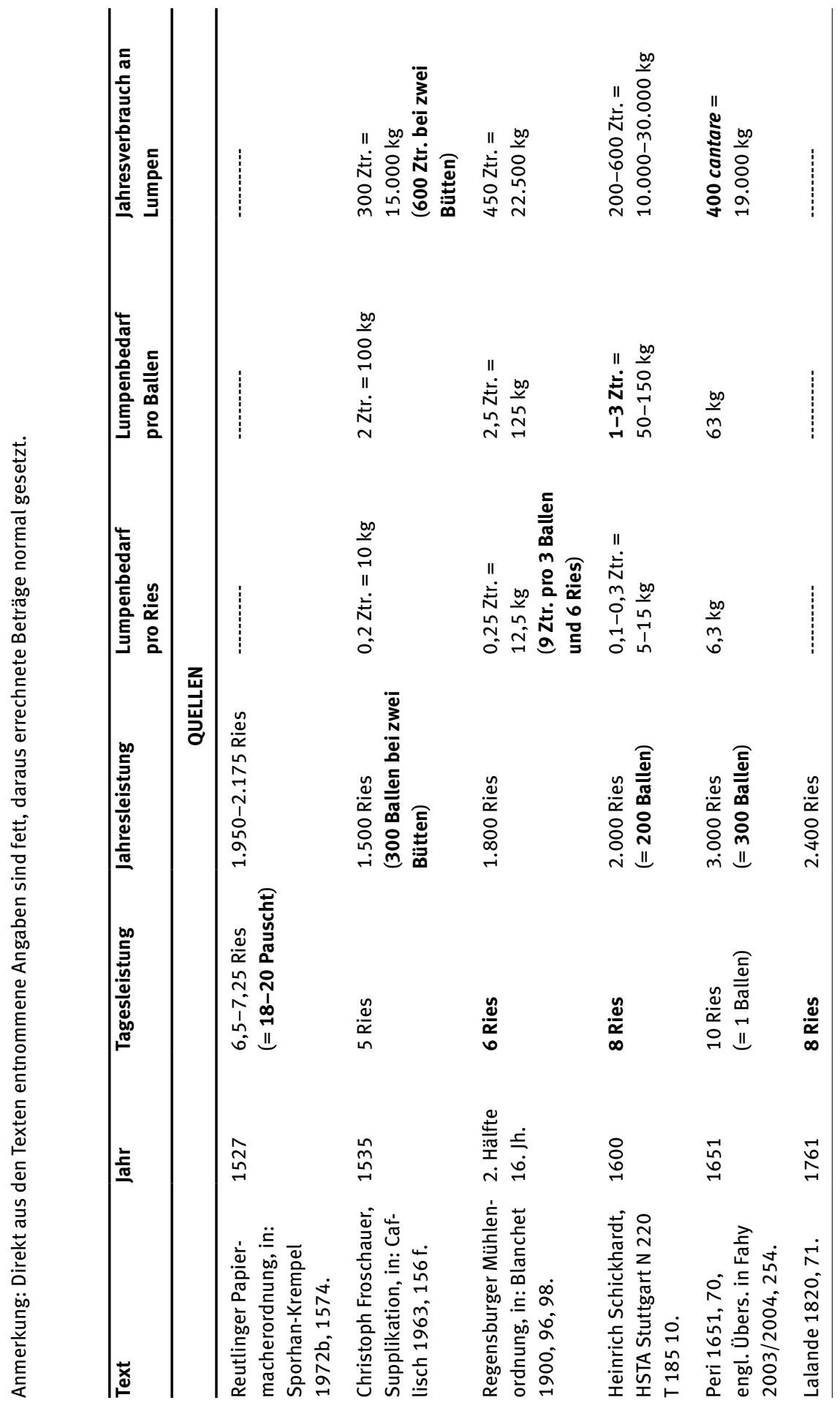




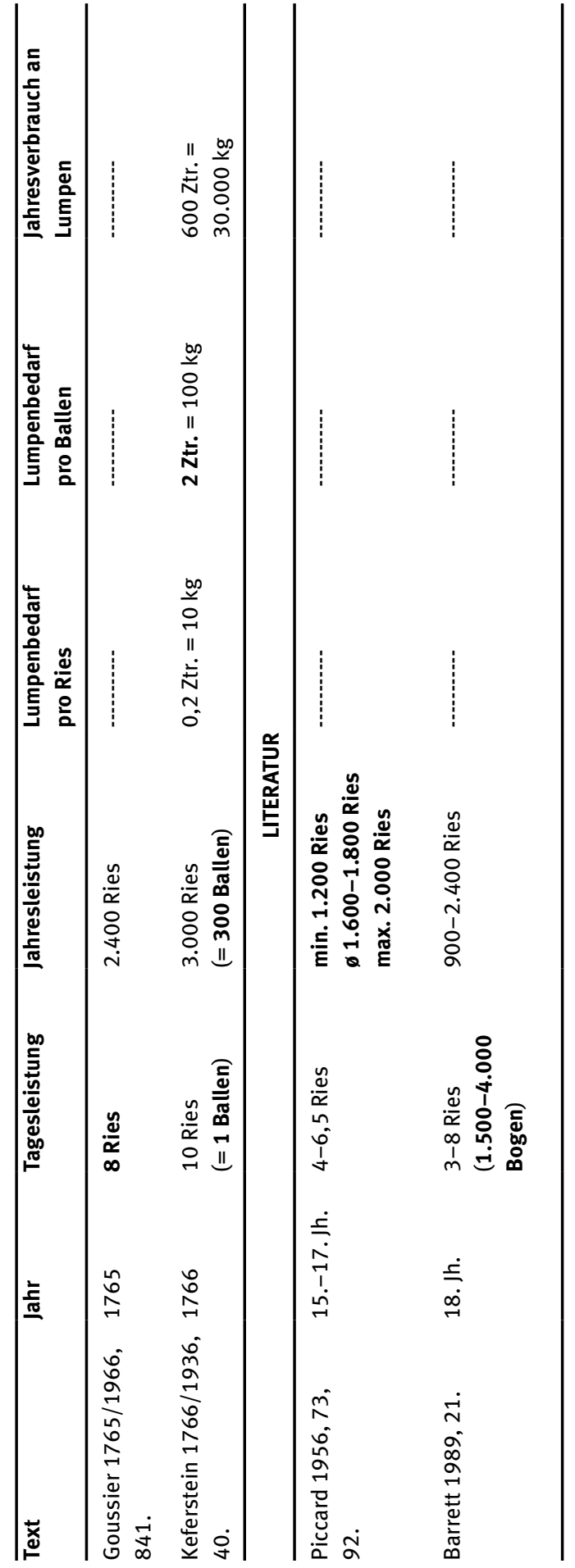




\subsection{Anhang III}

Analyse und Interpretation von Herstellungsspuren im Papier nach den Produktionsschritten 



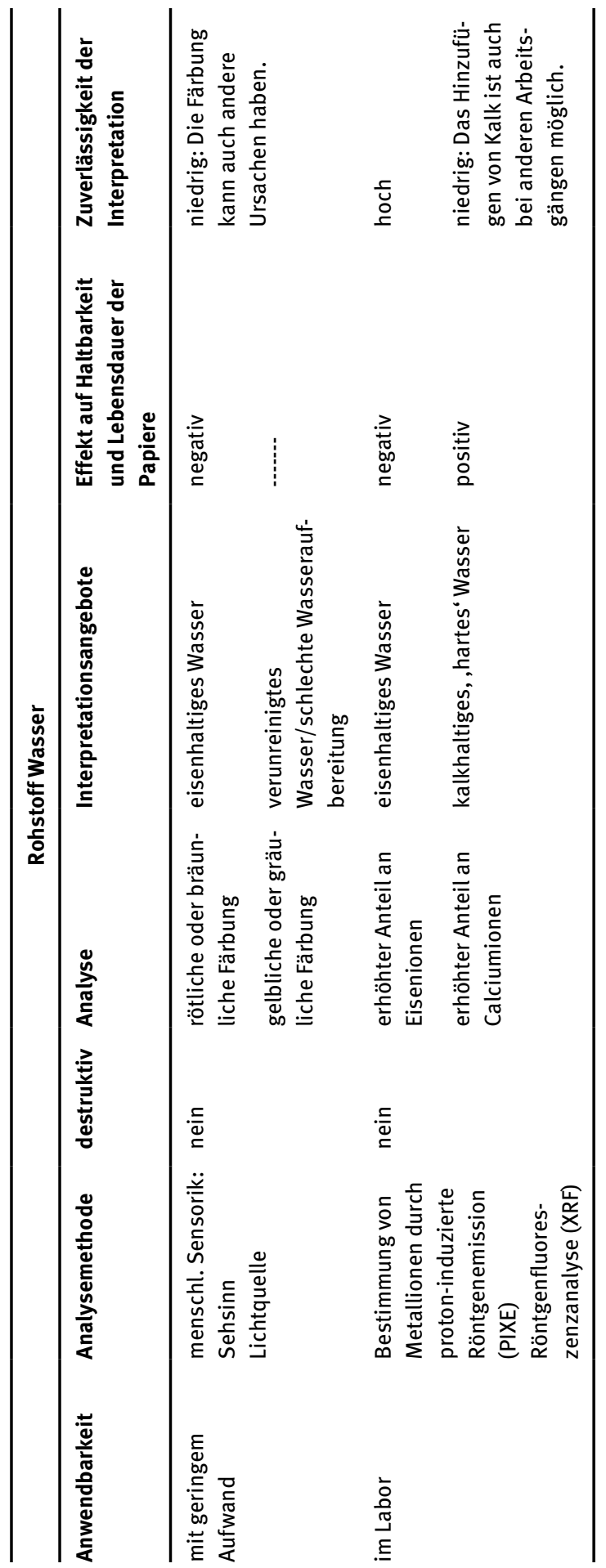




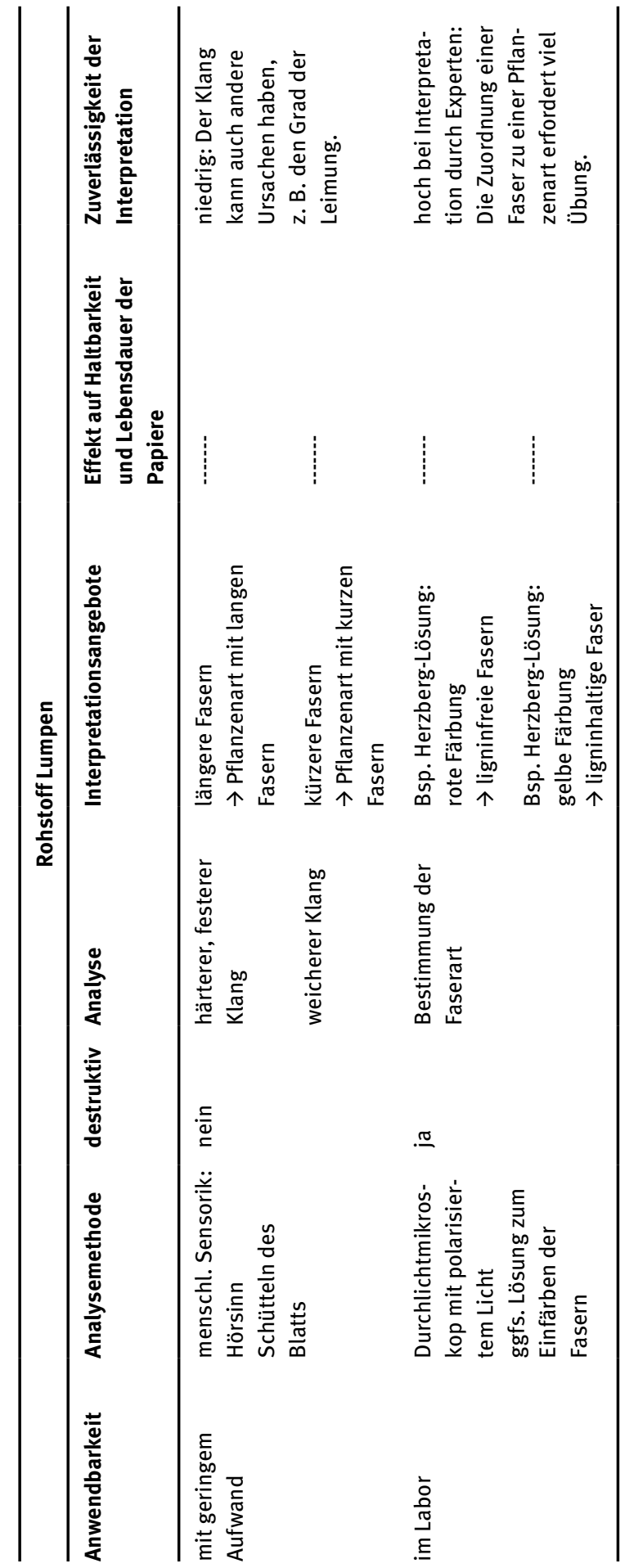




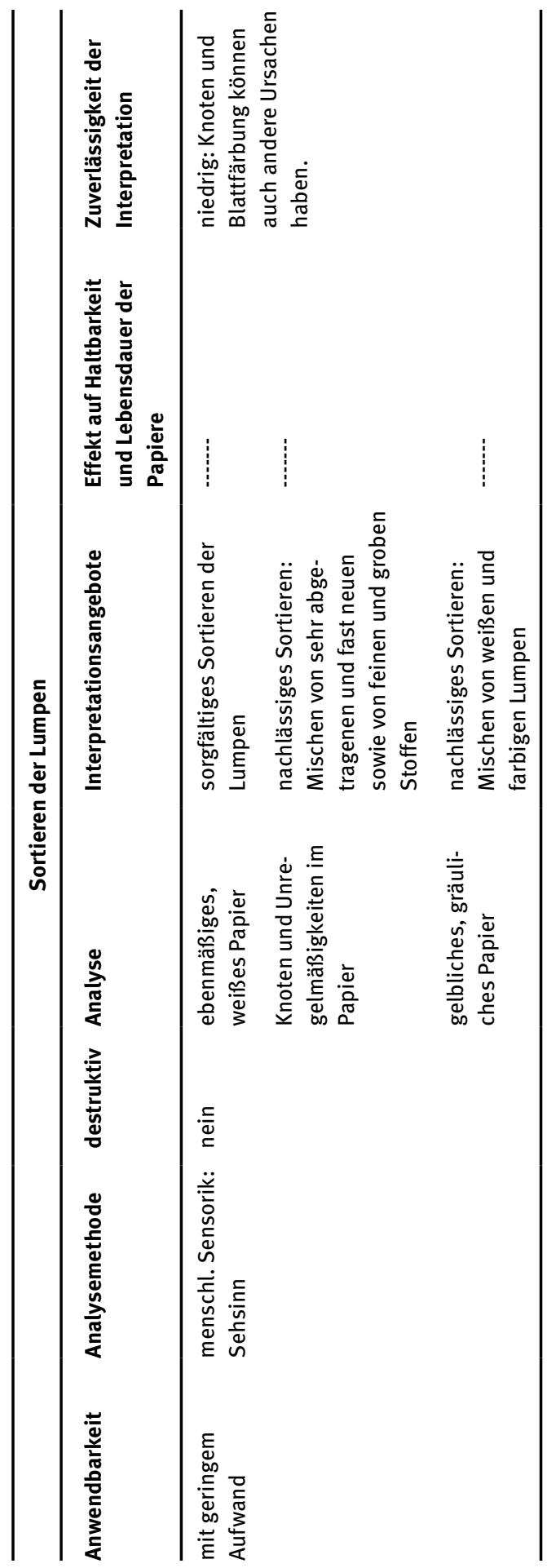




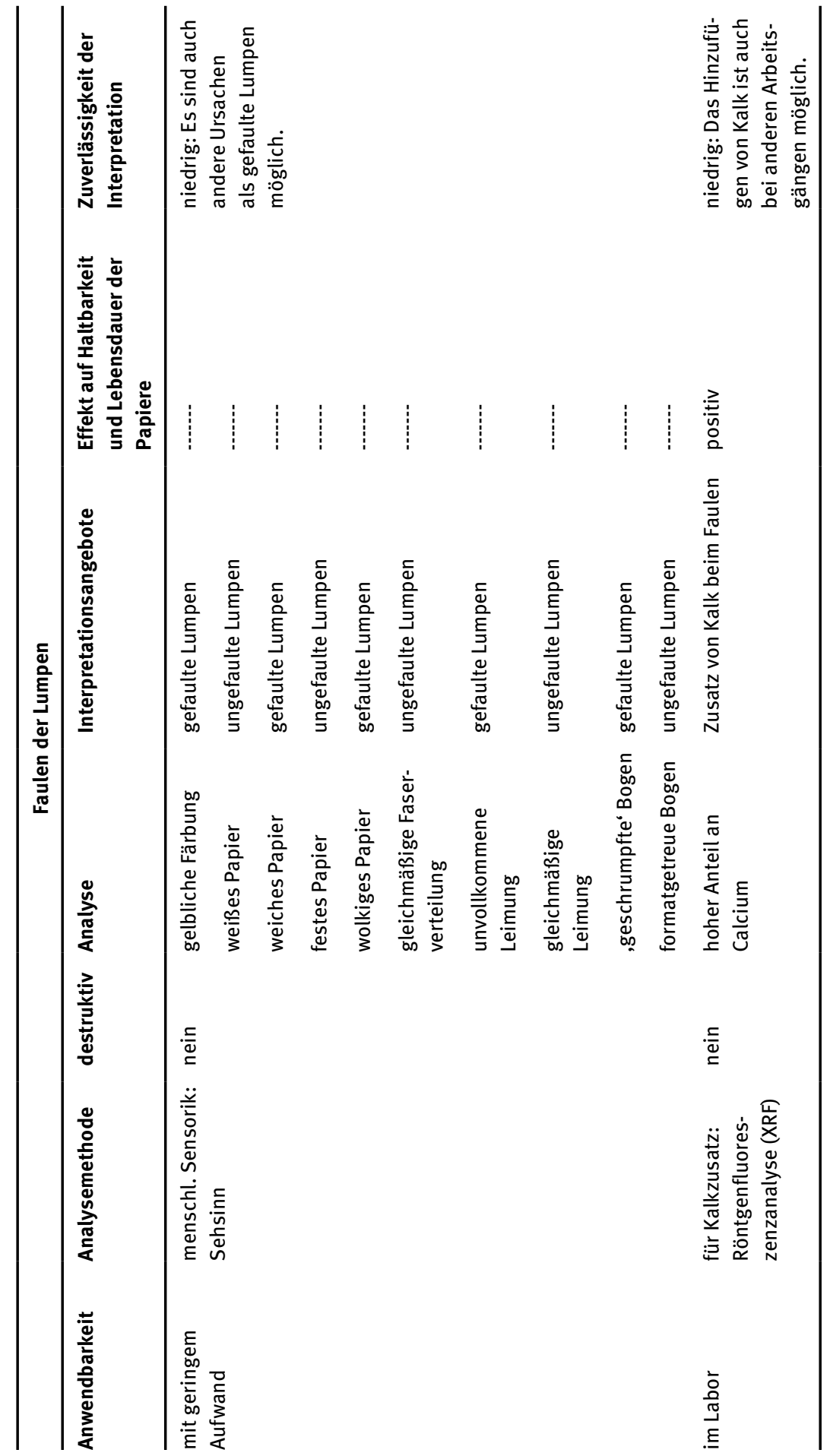




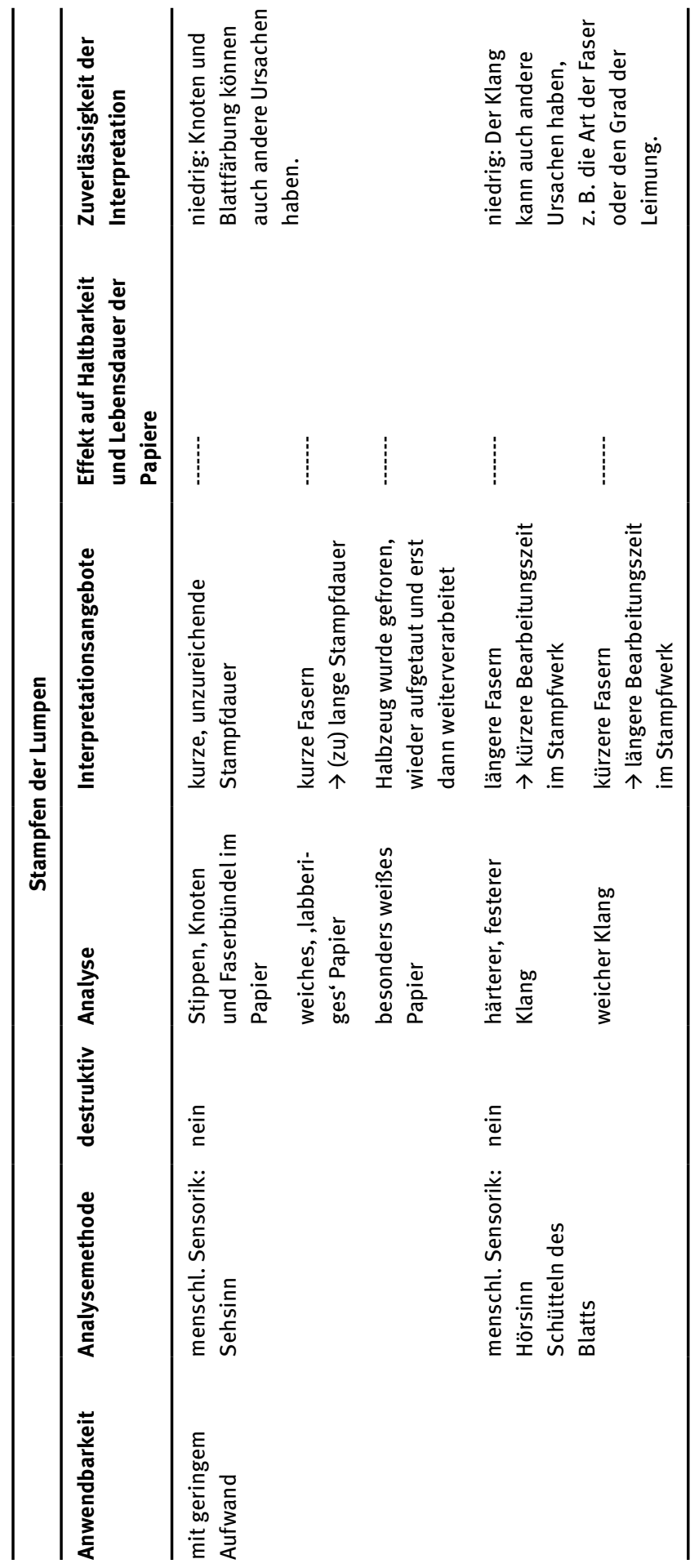




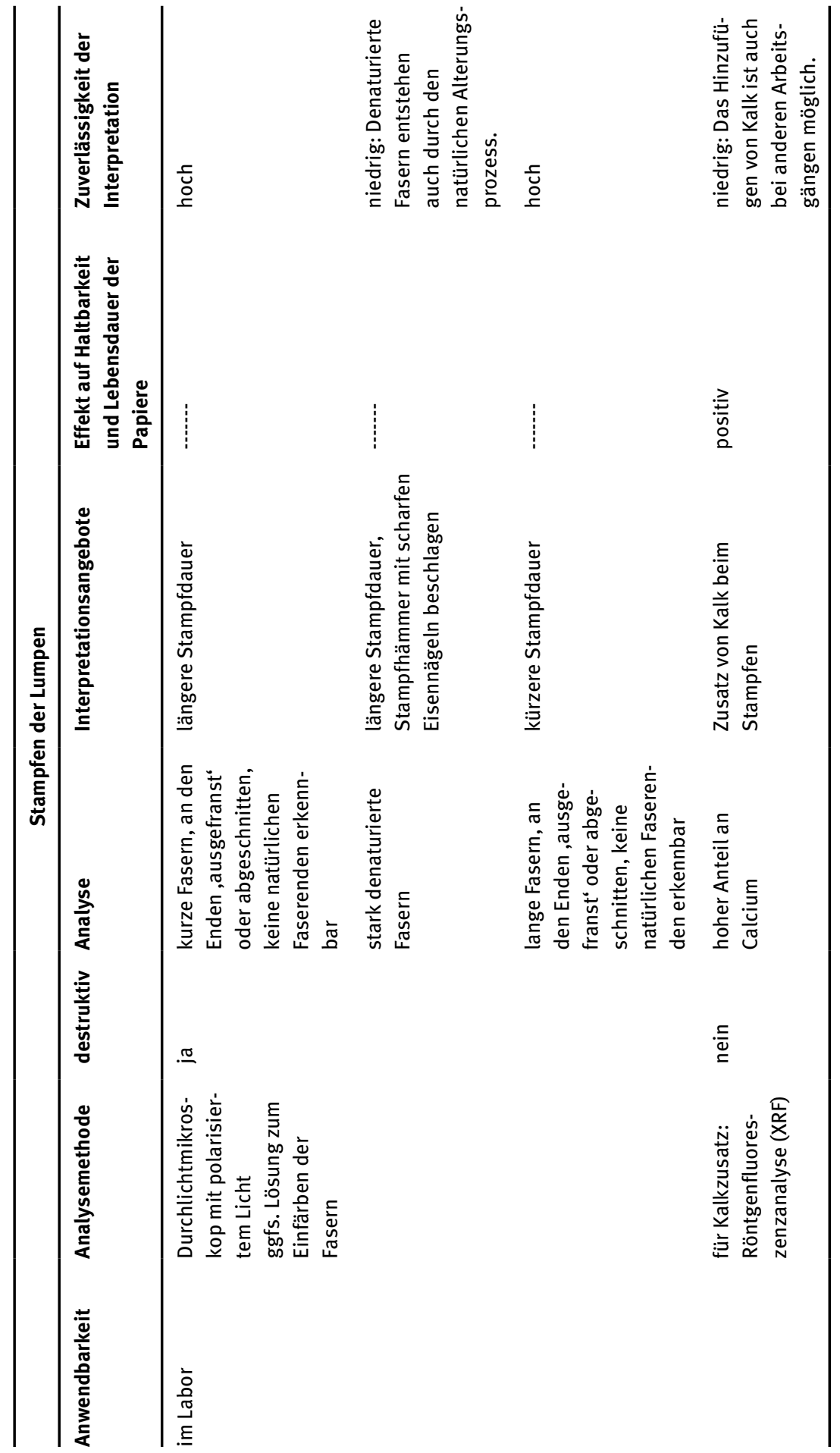




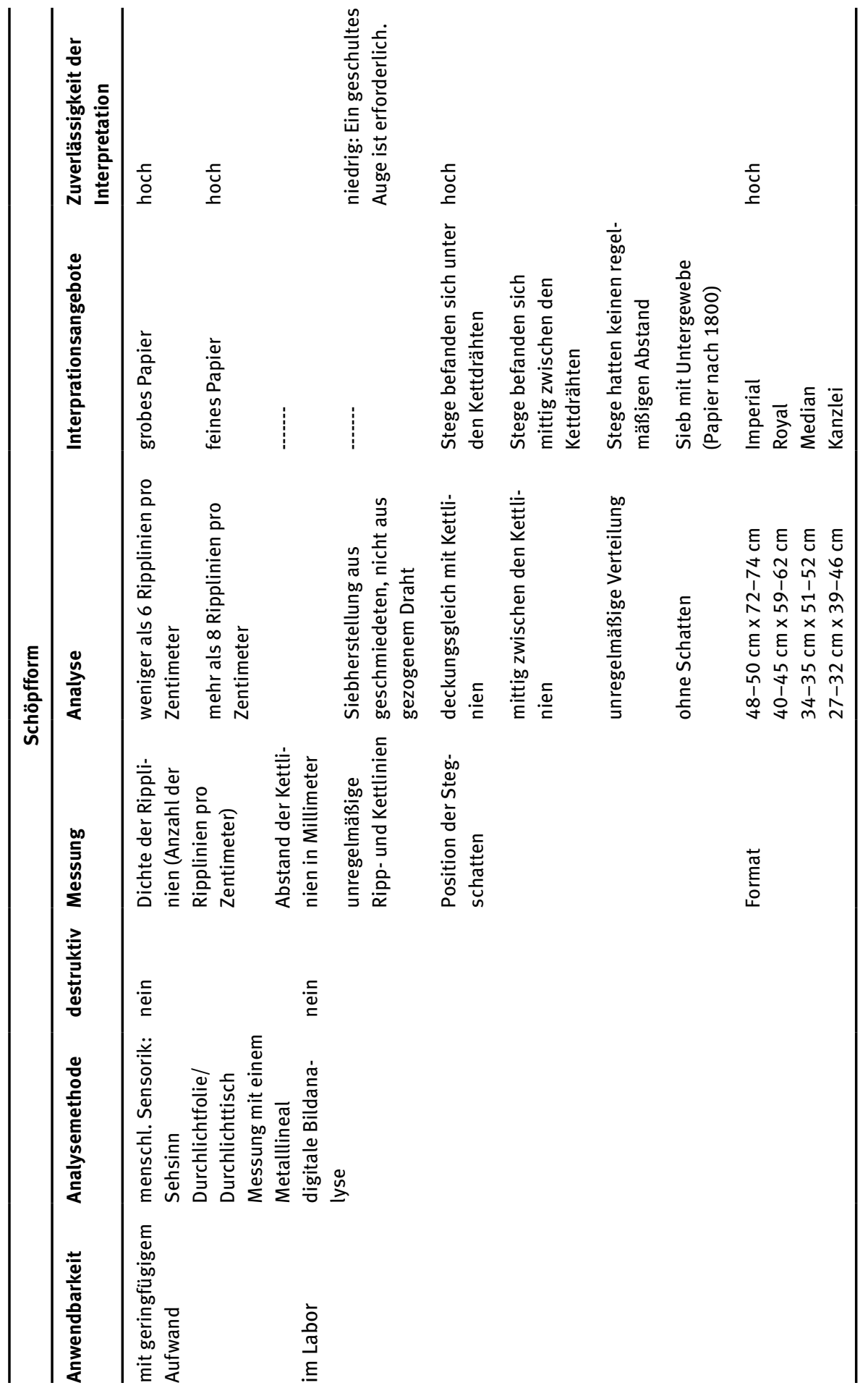




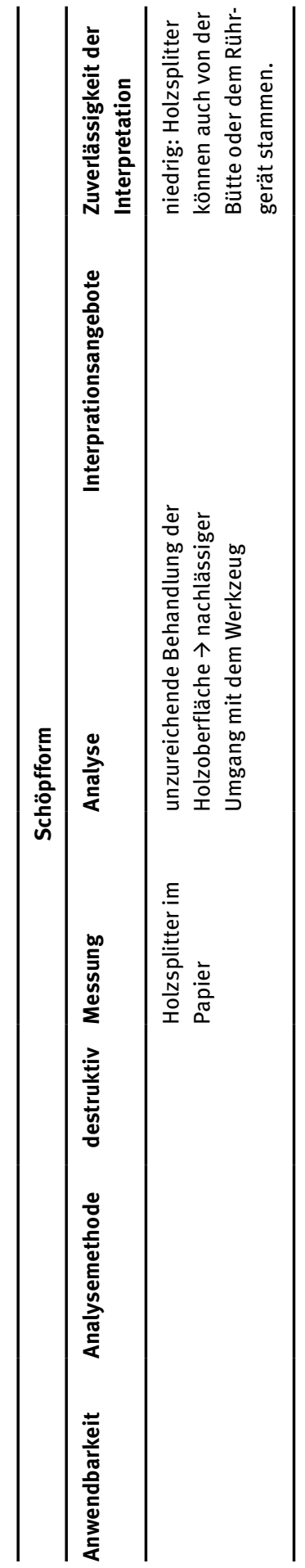




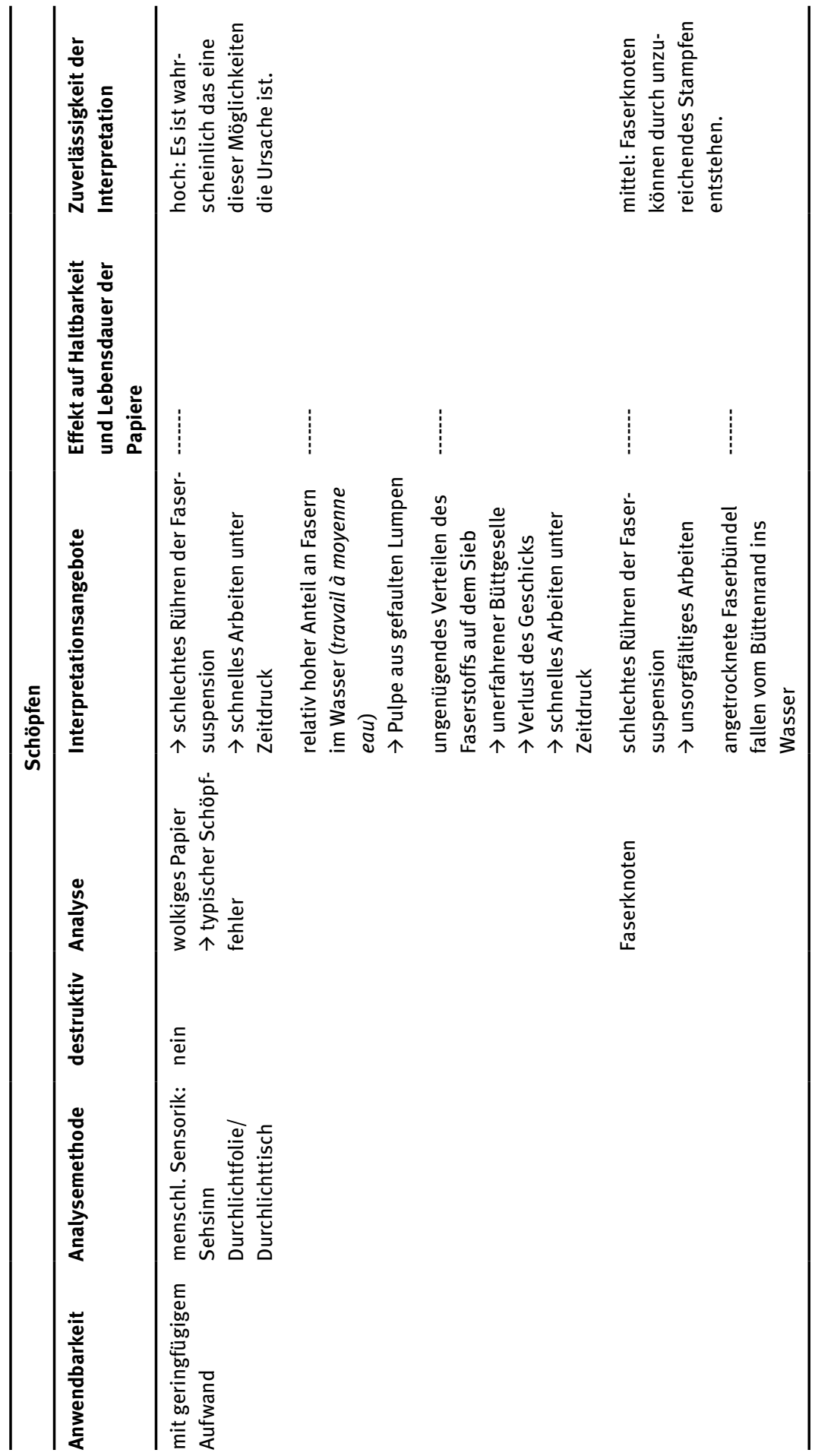




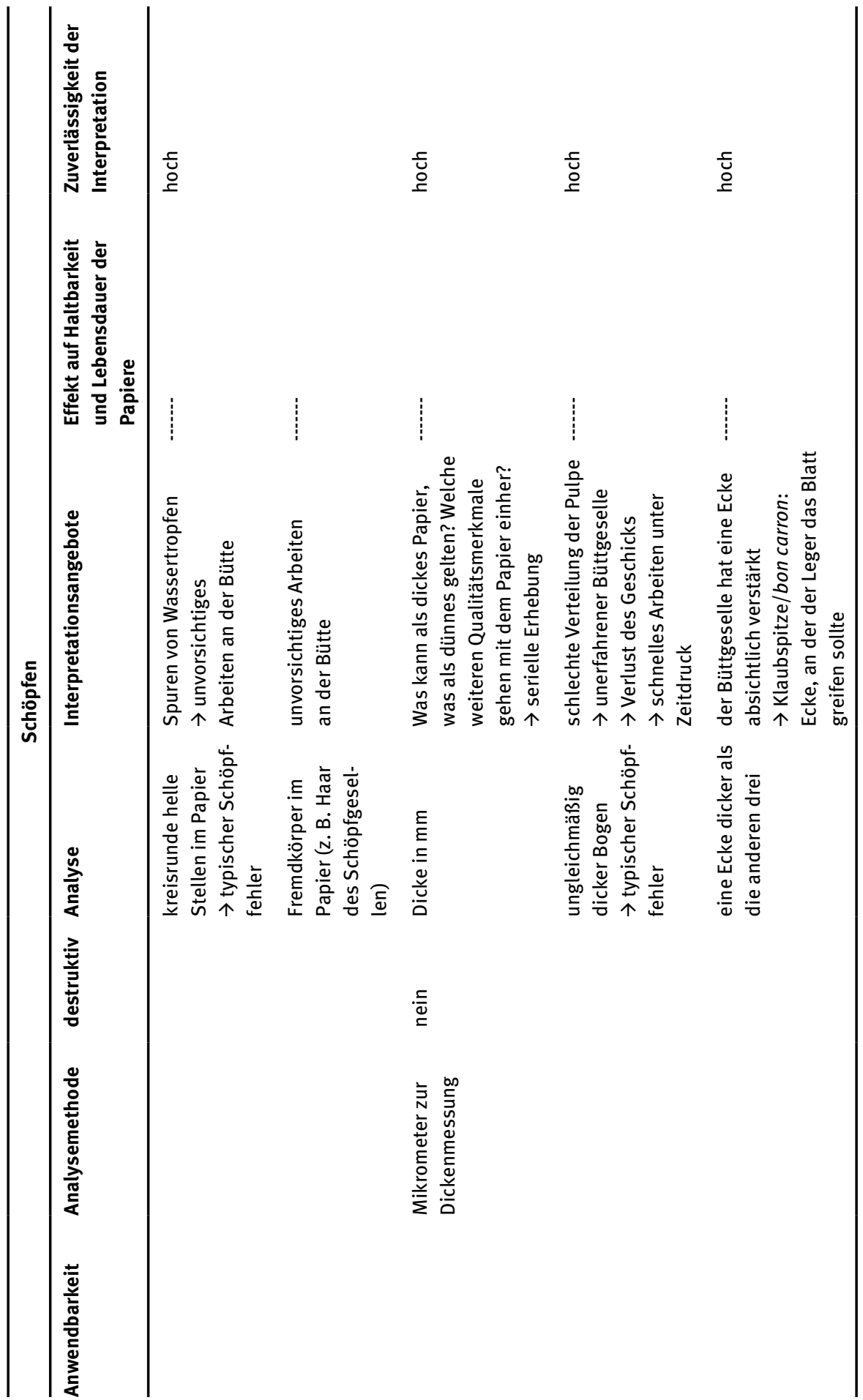




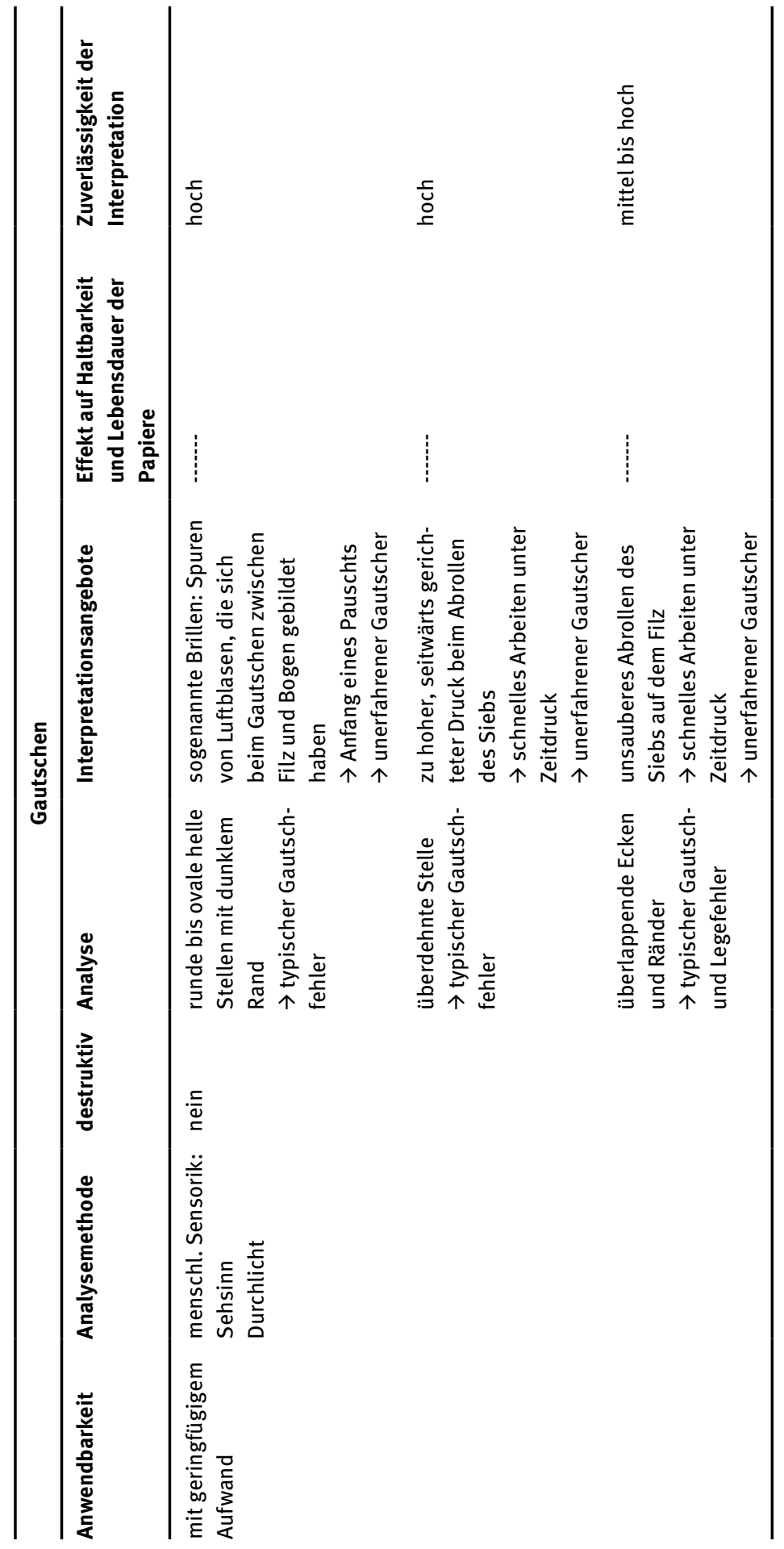




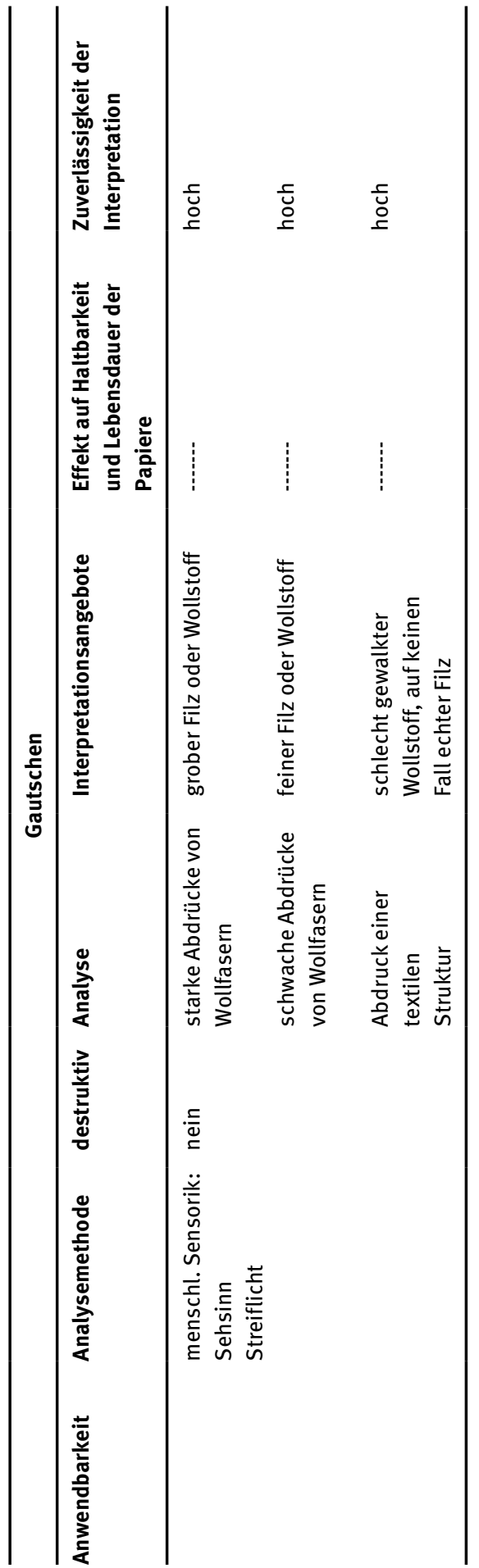




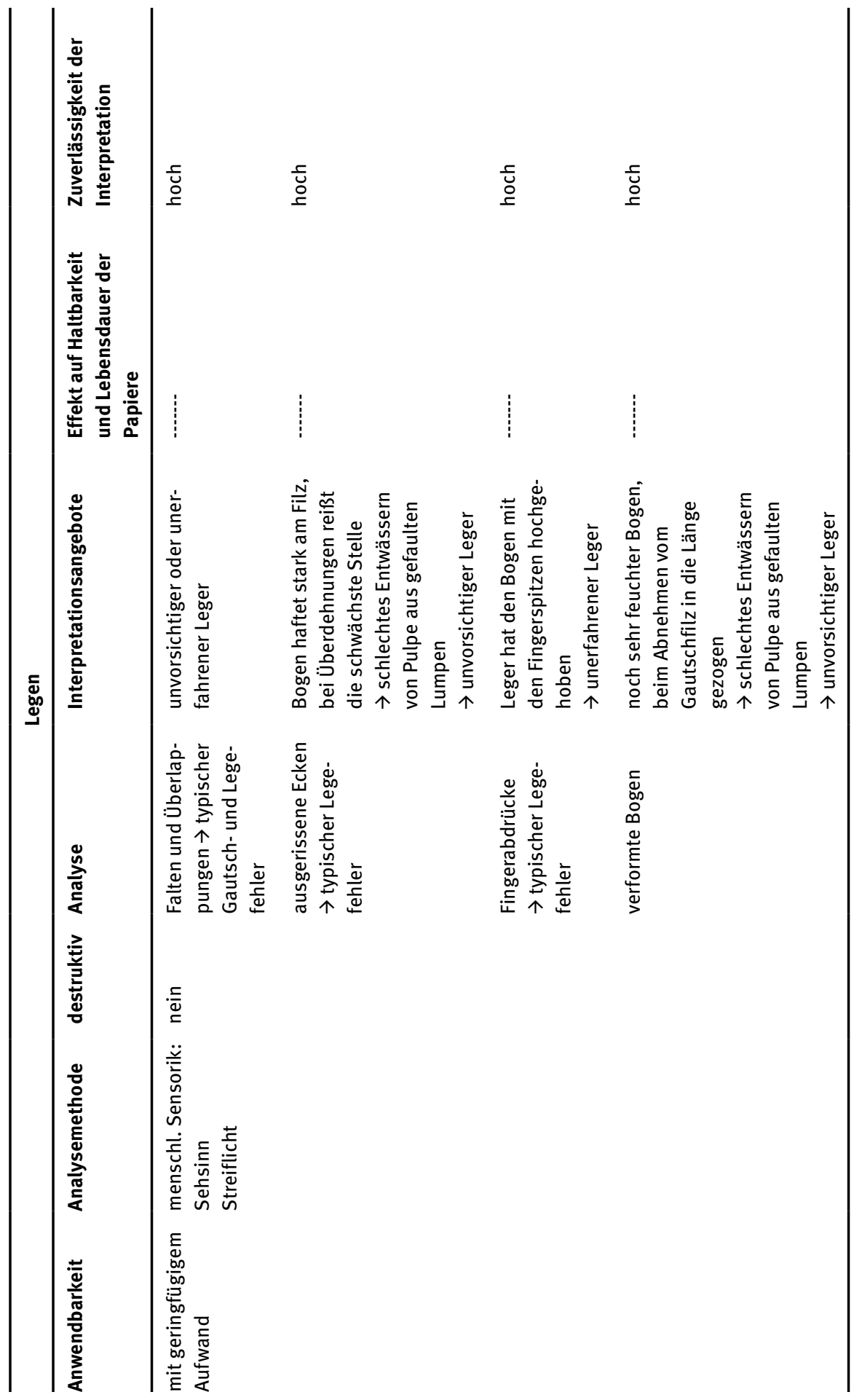




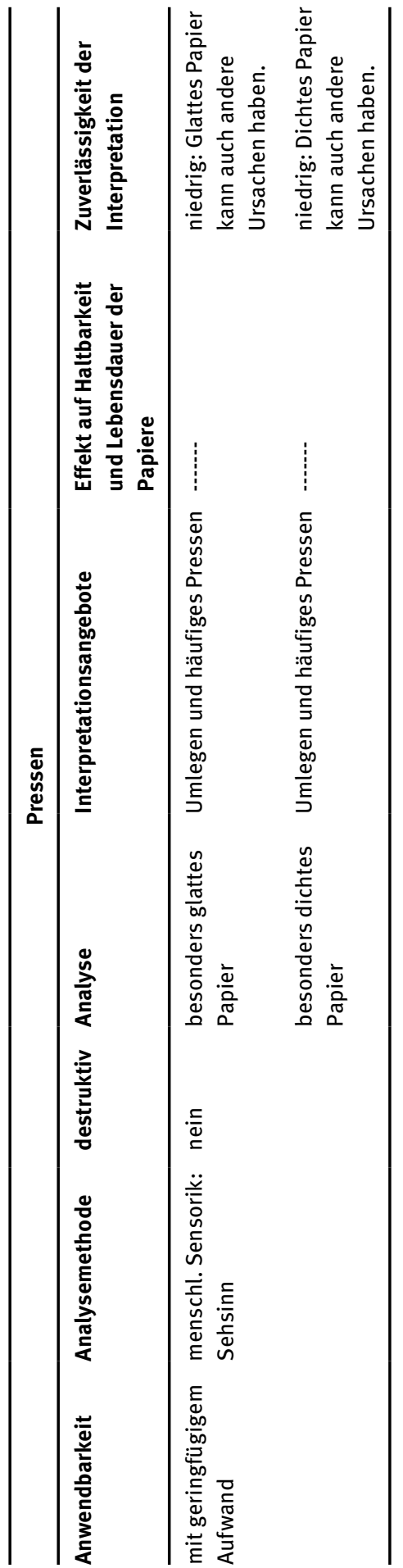




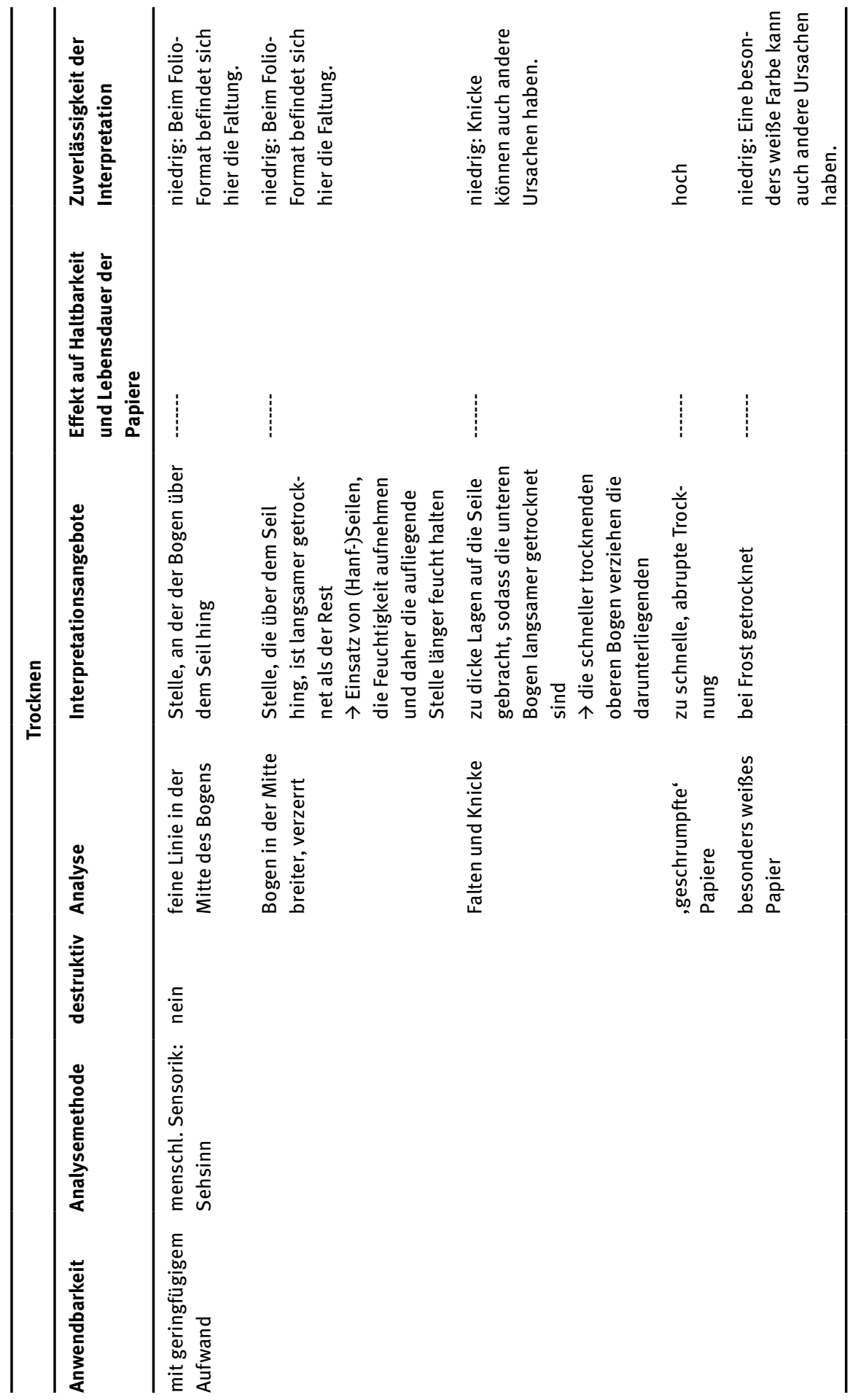




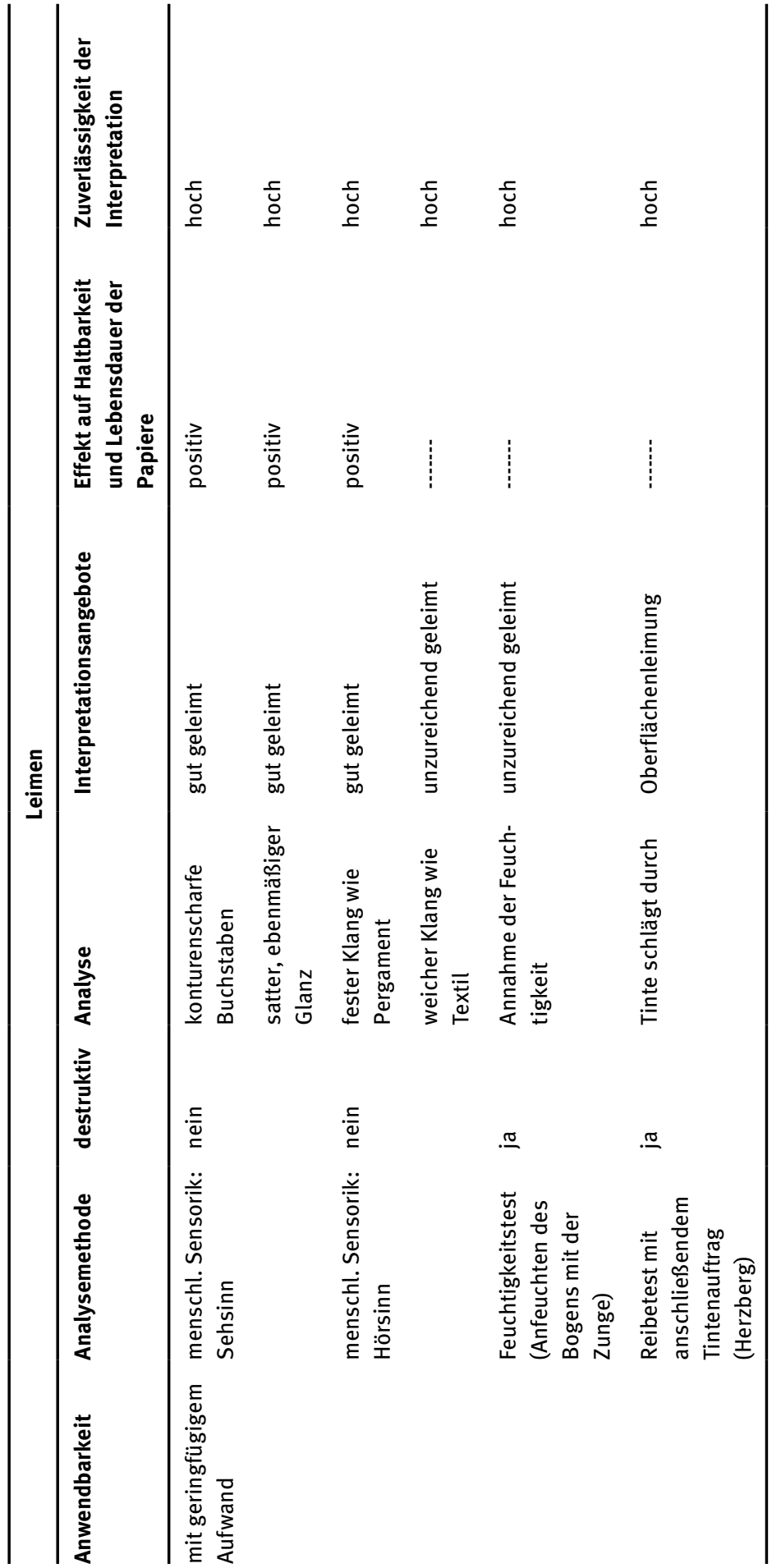




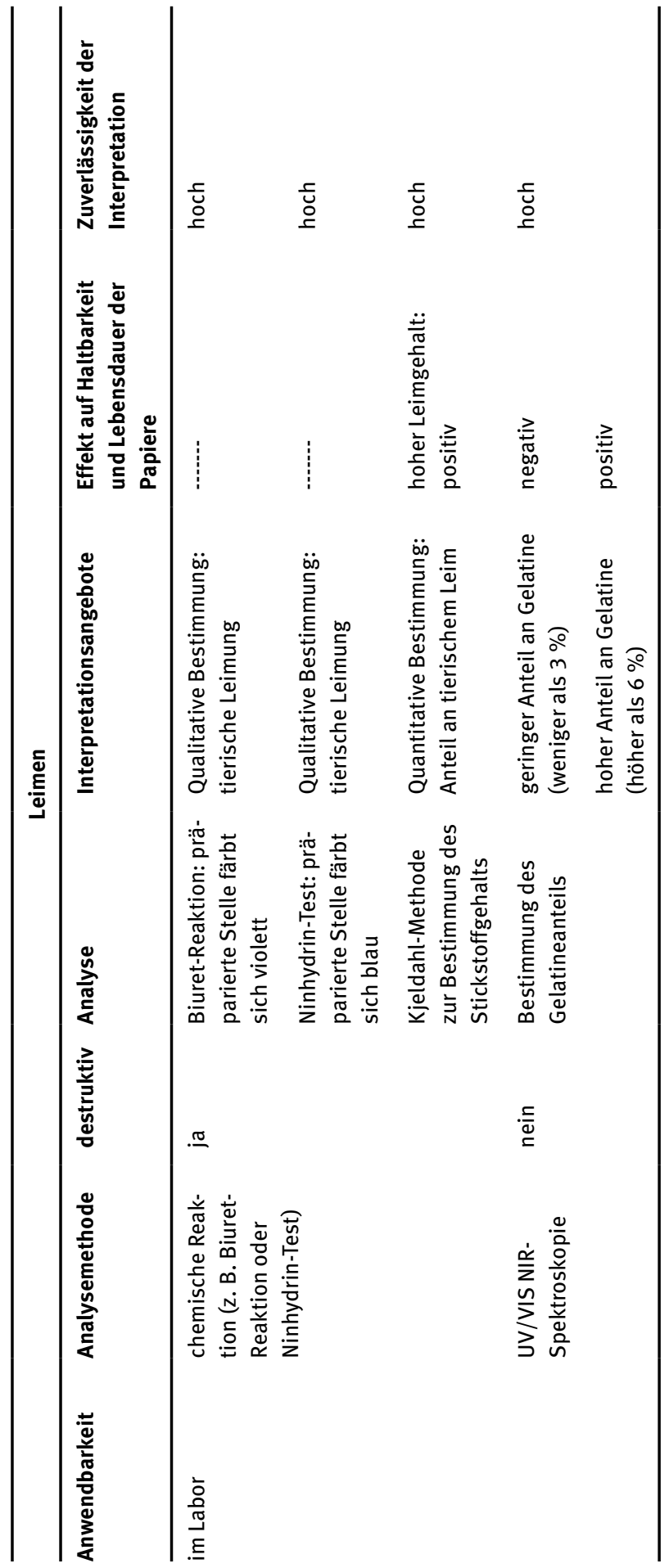




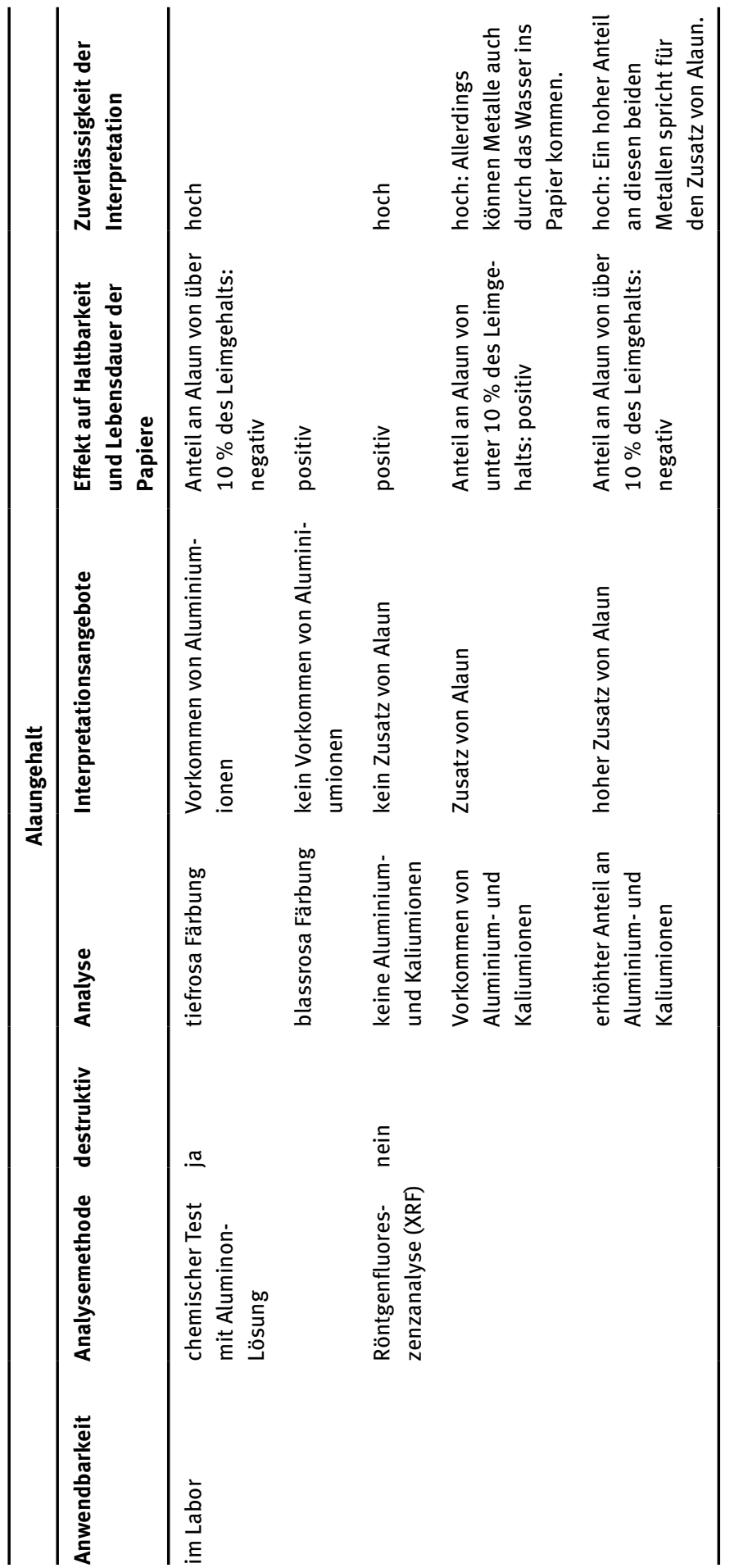




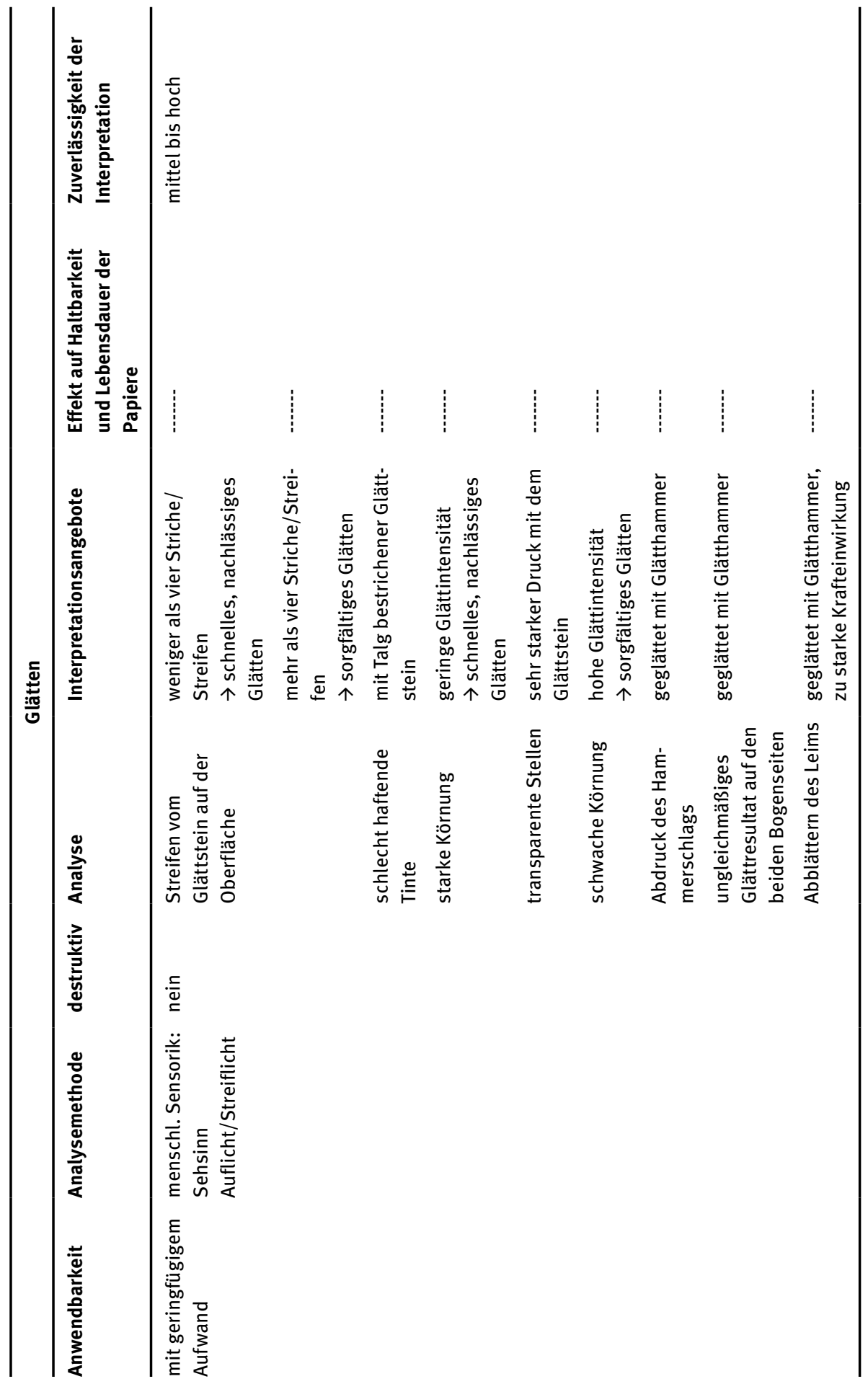




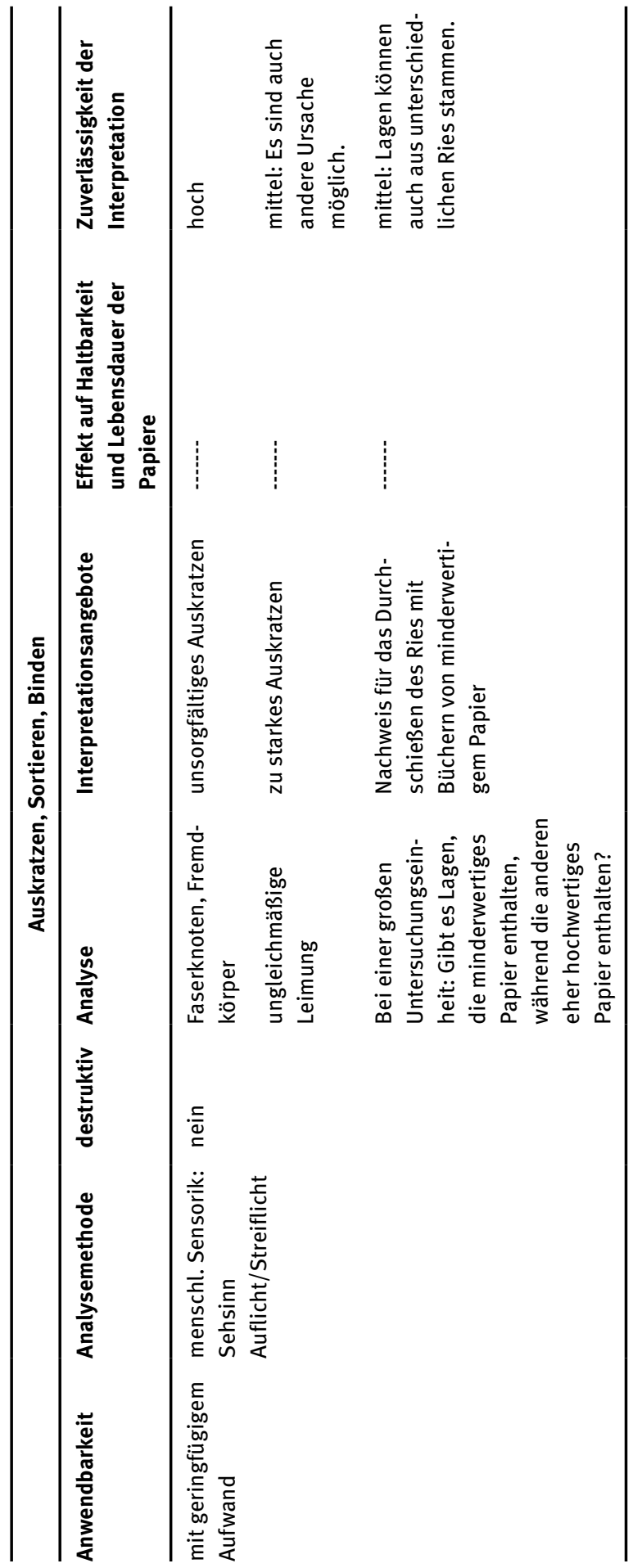




\subsection{Anhang IV}

Bausteine zu einem Beschreibungsformular für Herstellungsspuren in mittelalterlichen und frühneuzeitlichen Papieren 

Die hier in einem Baukasten zusammengestellten Bausteine für ein Beschreibungsformular vormoderner Papiere sollen dem Papierhistoriker das Rüstzeug zur Erfassung und Interpretation von Herstellungsspuren an die Hand geben. Dieser Baukasten wurde auf der Basis des IPH-Standards, des Beschreibungsprotokolls von Caroline Bourlet, Isabelle Bretthauer und Monique Zerdoun-Bat-Yehouda sowie des Formulars des Progetto Carta erstellt. ${ }^{3005}$ Insbesondere bei der Beschreibung der codicologischen sowie der inhaltlichen Einheit, der Siebstruktur, der Wasserzeichen und der Dickenmessung sowie für die Bilder und Schemata konnte auf diese Formulare zurückgegriffen werden. Weitere Kategorien wurden hinzugefügt, so beispielsweise die Oberflächenbeschaffenheit, die Haptik und die im Papier enthaltenen Fehler. Um nicht bei der Analyse der Papiere zu verharren, sondern um eine technikgeschichtliche Interpretation der erhobenen Daten zu ermöglichen, werden zu den verschiedenen Spuren außerdem Deutungsangebote gemacht. Die Erfassung der Herstellungsspuren im Archiv oder in der Bibliothek ist mit geringfügigem Aufwand und geringer technischer Ausrüstung zu leisten.

\section{Die codicologische Einheit ${ }^{3006}$}

\begin{tabular}{ll}
\hline Kategorie Daten \\
\hline
\end{tabular}

Aufbewahrungsort

Signatur

Bestand

Titel des Manuskripts

Datierung des Manuskripts

Auftraggeber (juristische

oder natürliche Person)

3005 Vgl. zum IPH-Standard P. Tschudin 2012a, Anhang II, 275-301. Zum Beschreibungsformular von Caroline Bourlet, Isabelle Bretthauer und Monique Zerdoun-Bat-Yehouda s. Bourlet/Bretthauer/Zerdoun Bat-Yehouda 2012/2017; zum Progetto Carta vgl. Ornato et al. 2001, Bd. 1, 44, 77-84.

3006 Vgl. Bourlet/Bretthauer/Zerdoun Bat-Yehouda 2012/2017, 1. 


\begin{tabular}{ll}
\hline Kategorie & Daten \\
\hline Zusammensetzung des & $\square$ zusammengesetztes Manuskript \\
Manuskripts & $\square$ homogenes Manuskript \\
& bei homogenem Manuskript: weiße Hefte oder Blätter am Ende \\
& $\square$ nein $\square$ ja Anzahl: $\square$ \\
Einband & $\square$ zeitgenössischer Einband \\
& $\square$ Holzeinband $\square$ weicher Einband \\
& $\square$ Leder $\square$ Pergament $\square$ Makulatur \\
& $\square$ Sonstiges:
\end{tabular}

\section{Die inhaltliche Einheit ${ }^{3007}$}

\subsection{Text}

Kategorie Daten

Titel/Inhalt des Texts

Entstehungszeit des Texts

Gattung

Kommentare zum Inhalt

\subsection{Materielle Form}

\begin{tabular}{ll}
\hline Kategorie & Daten \\
\hline Zusammensetzung & $\square$ Papier $\square$ Papier/Pergament \\
& $\square$ Anzahl Pergamentblätter:
\end{tabular}

Anzahl der Blätter im Doku-

ment

Anzahl der Lagen

3007 Vgl. Bourlet/Bretthauer/Zerdoun Bat-Yehouda 2012/2017, 1. 


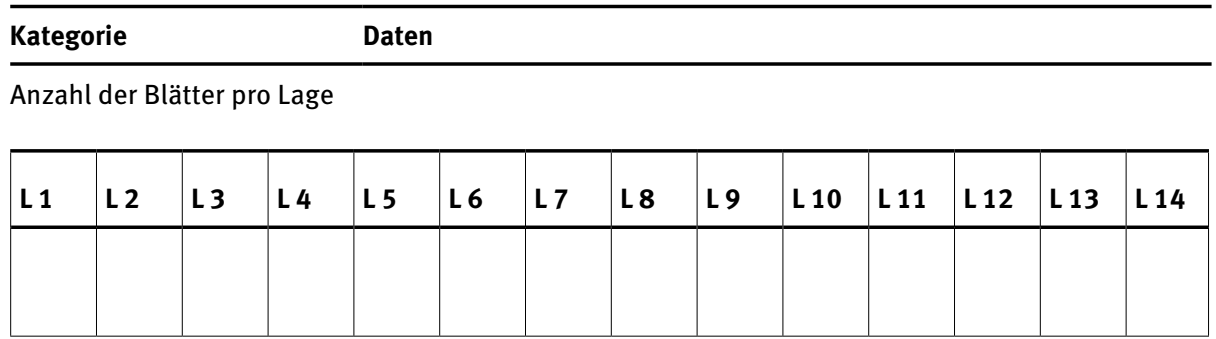

Format des Dokuments

$$
\begin{aligned}
& \square \text { ganzer Bogen } \\
& \square \text { Folio (2 Blätter, 4 Seiten) } \\
& \square \text { Quart (4 Blätter, 8 Seiten) } \\
& \square \text { Oktav (8 Blätter, 16 Seiten) } \\
& \square \text { Duodez (12 Blätter, } 24 \text { Seiten) }
\end{aligned}
$$

Richtung der Ripplinien $\quad \square$ horizontal $\square$ vertikal

Richtung der Kettlinien $\quad \square$ horizontal $\square$ vertikal

Anzahl der Wasserzeichen-

motive

\section{Papierbogen bzw. Doppelblatt}

\subsection{Allgemeine Angaben zum Bogen ${ }^{3008}$}

\begin{tabular}{ll}
\hline Kategorie & Daten \\
\hline $\begin{array}{l}\text { gewähltes Doppelblatt } \\
\text { (Foliierung /Paginierung) }\end{array}$ & \\
Wasserzeichenmotiv & \\
Erhaltungszustand & $\square$ hervorragend $\square$ gut $\square$ mittel \\
& $\square$ schlecht (Feuchtigkeit) $\square$ schlecht (zerrissen) \\
& $\square$ Flecken $\square$ Löcher \\
& $\square$ restauriert \\
\hline
\end{tabular}

3008 Vgl. Bourlet/Bretthauer/Zerdoun Bat-Yehouda 2012/2017, 2. 


\section{$3.2 \mathrm{Größe} e^{3009}$}

\begin{tabular}{|c|c|c|}
\hline Kategorie & Analyse \& Interpretationsmöglichkeiten & Arbeitsschritt \\
\hline $\begin{array}{l}\text { Größe des } \\
\text { Doppelblatts }\end{array}$ & 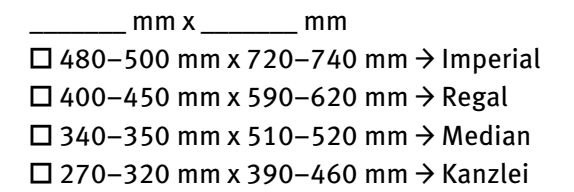 & Schöpfen \\
\hline Beschnitt & $\begin{array}{l}\square \text { nein } \square \text { ja } \\
\text { Blatt 1:___ } \square \text { Kopf } \square \text { Ende } \square \text { lange Seite } \\
\text { Blatt 2:___ } \square \text { Kopf } \square \text { Ende } \square \text { lange Seite } \\
\square \text { Fragment }\end{array}$ & \\
\hline \multirow[t]{2}{*}{$\square$ formatgetreue Bogen } & ungefaulte Lumpen & $\begin{array}{l}\text { Rohstoffaufbereitung: } \\
\text { Faulen }\end{array}$ \\
\hline & langsames Trocknen & Trocknen \\
\hline \multirow[t]{2}{*}{$\begin{array}{l}\square \text { verformte } \\
\text { Bogen }\end{array}$} & $\begin{array}{l}\text { noch sehr feuchter Bogen, der beim Abneh- } \\
\text { men vom Gautschfilz in die Länge gezogen } \\
\text { wurde }\end{array}$ & Legen \\
\hline & $\begin{array}{l}\text { schlechtes Entwässern von Pulpe aus gefaul- } \\
\text { ten Lumpen }\end{array}$ & $\begin{array}{l}\text { Rohstoffaufbereitung: } \\
\text { Faulen }\end{array}$ \\
\hline \multirow[t]{2}{*}{$\square$,geschrumpfte‘ Bogen } & gefaulte Lumpen & $\begin{array}{l}\text { Rohstoffaufbereitung: } \\
\text { Faulen }\end{array}$ \\
\hline & zu schnelles Trocknen & Trocknen \\
\hline
\end{tabular}

\subsection{Farbe}

\begin{tabular}{lll}
\hline Farbe & Interpretationsmöglichkeiten & Arbeitsschritt \\
\hline$\square$ helles Cremeweiß & \\
$\square$ dunkles Cremeweiß &
\end{tabular}

3009 Vgl. Bourlet/Bretthauer/Zerdoun Bat-Yehouda 2012/2017, 2. 


\begin{tabular}{|c|c|c|}
\hline Farbe & Interpretationsmöglichkeiten & Arbeitsschritt \\
\hline \multirow[t]{4}{*}{$\square$ sehr weiß } & $\begin{array}{l}\text { sorgfältiges Sortieren der Lumpen: Verwen- } \\
\text { dung ausschließlich von weißen Leinenlum- } \\
\text { pen }\end{array}$ & $\begin{array}{l}\text { Rohstoffaufbereitung: } \\
\text { Sortieren der Lumpen }\end{array}$ \\
\hline & Papier aus ungefaulten Lumpen & $\begin{array}{l}\text { Rohstoffaufbereitung: } \\
\text { Faulen }\end{array}$ \\
\hline & $\begin{array}{l}\text { Halbzeug wurde gefroren, wieder aufgetaut } \\
\text { und erst dann weiterverarbeitet }\end{array}$ & $\begin{array}{l}\text { Rohstoffaufbereitung: } \\
\text { Stampfen }\end{array}$ \\
\hline & bei Frost getrocknet & Trocknen \\
\hline$\square$ rötlich & verwendetes Wasser eisenhaltig & Rohstoff Wasser \\
\hline$\square$ bräunlich & verwendetes Wasser eisenhaltig & Rohstoff Wasser \\
\hline \multirow[t]{3}{*}{$\square$ gelblich } & $\begin{array}{l}\text { verunreinigtes Wasser/schlechte Wasserauf- } \\
\text { bereitung }\end{array}$ & Rohstoff Wasser \\
\hline & $\begin{array}{l}\text { unsorgfältiges Sortieren: Mischen von weißen } \\
\text { und farbigen Lumpen }\end{array}$ & $\begin{array}{l}\text { Rohstoffaufbereitung: } \\
\text { Sortieren der Lumpen }\end{array}$ \\
\hline & Papier aus gefaulten Lumpen & $\begin{array}{l}\text { Rohstoffaufbereitung: } \\
\text { Faulen }\end{array}$ \\
\hline \multirow[t]{2}{*}{$\square$ gräulich } & $\begin{array}{l}\text { verunreinigtes Wasser/schlechte Wasserauf- } \\
\text { bereitung }\end{array}$ & Rohstoff Wasser \\
\hline & $\begin{array}{l}\text { unsorgfältiges Sortieren: Mischen von weißen } \\
\text { und farbigen Lumpen }\end{array}$ & $\begin{array}{l}\text { Rohstoffaufbereitung: } \\
\text { Sortieren der Lumpen }\end{array}$ \\
\hline
\end{tabular}

\subsection{Papieroberfläche}

\begin{tabular}{|c|c|c|}
\hline Oberflächenstruktur & Interpretationsmöglichkeiten & Arbeitsschritt \\
\hline $\begin{array}{l}\square \text { starke Abdrücke von } \\
\text { Wollfasern }\end{array}$ & grober Filz oder Wollstoff & Gautschen \\
\hline $\begin{array}{l}\square \text { schwache Abdrücke } \\
\text { von Wollfasern }\end{array}$ & feiner Filz oder Wollstoff & Gautschen \\
\hline $\begin{array}{l}\square \text { Abdruck einer textilen } \\
\text { Struktur }\end{array}$ & $\begin{array}{l}\text { schlecht gewalkter Wollstoff, auf keinen Fall } \\
\text { echter Filz }\end{array}$ & Gautschen \\
\hline$\square$ konturenscharfe & gute Leimung & Leimen \\
\hline Buchstaben & $\rightarrow$ event. ungefaulte Lumpen & $\begin{array}{l}\text { Rohstoffaufbereitung: } \\
\text { Faulen }\end{array}$ \\
\hline$\square$ fließende Buchstaben & $\begin{array}{l}\text { schlechte Leimung } \\
\rightarrow \text { event. gefaulte Lumpen }\end{array}$ & $\begin{array}{l}\text { Leimen } \\
\text { Rohstoffaufbereitung: } \\
\text { Faulen }\end{array}$ \\
\hline
\end{tabular}




\begin{tabular}{|c|c|c|}
\hline Oberflächenstruktur & Interpretationsmöglichkeiten & Arbeitsschritt \\
\hline $\begin{array}{l}\square \text { satter, ebenmäßiger } \\
\text { Glanz }\end{array}$ & $\begin{array}{l}\text { gute, gleichmäßige Leimung } \\
\rightarrow \text { event. ungefaulte Lumpen }\end{array}$ & $\begin{array}{l}\text { Leimen } \\
\text { Rohstoffaufbereitung: } \\
\text { Faulen }\end{array}$ \\
\hline $\begin{array}{l}\square \text { unregelmäßiger } \\
\text { Glanz, matte Stellen }\end{array}$ & $\begin{array}{l}\text { Abblättern des Leims durch Glätten mit Glätt- } \\
\text { hammer unter zu starker Krafteinwirkung }\end{array}$ & Glätten \\
\hline \multirow[t]{2}{*}{$\begin{array}{l}\square \text { Streifen vom Glätt- } \\
\text { stein auf der Oberfläche }\end{array}$} & $\begin{array}{l}\square \text { weniger als vier Striche/Streifen } \\
\rightarrow \text { schnelles, unsorgfältiges Glätten }\end{array}$ & Glätten \\
\hline & $\begin{array}{l}\square \text { mehr als vier Striche/Streifen } \\
\rightarrow \text { sorgfältiges Glätten }\end{array}$ & Glätten \\
\hline $\begin{array}{l}\square \text { schlecht haftende } \\
\text { Tinte }\end{array}$ & mit Talg bestrichener Glättstein & Glätten \\
\hline$\square$ starke Körnung & geringe Glättintensität & Glätten \\
\hline$\square$ transparente Stellen & sehr starker Druck mit dem Glättstein & Glätten \\
\hline$\square$ schwache Körnung & hohe Glättintensität & Glätten \\
\hline $\begin{array}{l}\square \text { Abdruck des Hammer- } \\
\text { schlags }\end{array}$ & geglättet mit Glätthammer & Glätten \\
\hline $\begin{array}{l}\square \text { ungleichmäßiges } \\
\text { Glättresultat auf den } \\
\text { beiden Bogenseiten }\end{array}$ & geglättet mit Glätthammer & Glätten \\
\hline
\end{tabular}

\subsection{Klang (Schütteltest)}

\begin{tabular}{lll}
\hline Klang & Interpretationsmöglichkeiten & Arbeitsschritt \\
\hline$\square$ harter, fester Klang & Pflanzenart mit langen Fasern & Rohstoff Lumpen \\
& kürzere Bearbeitungszeit im Stampfwerk & $\begin{array}{l}\text { Rohstoffaufbereitung: } \\
\text { Stampfen }\end{array}$ \\
& gute Leimung & Leimen \\
$\square$ weicher Klang & Pflanzenart mit kurzen Fasern & Rohstoff Lumpen \\
& längere Bearbeitungszeit im Stampfwerk & Rohstoffaufbereitung: \\
& & Stampfen \\
& schlechte Leimung & Leimen \\
\hline
\end{tabular}




\subsection{Haptik}

\begin{tabular}{lll}
\hline Haptik & Interpretationsmöglichkeiten & Arbeitsschritt \\
\hline$\square$ weiches Papier & gefaulte Lumpen & Rohstoffaufbereitung: \\
& kuulen & Rohstoffaufbereitung: \\
& $\rightarrow(\mathrm{zu})$ lange Stampfdauer & Stampfen \\
& geringe Leimung & Leimen \\
$\square$ festes Papier & ungefaulte Lumpen & Rohstoffaufbereitung: \\
& & Faulen \\
& lange Fasern & Rohstoffaufbereitung: \\
& $\rightarrow$ kürzere Stampfdauer & Stampfen \\
& gute Leimung & Leimen \\
\hline
\end{tabular}

\subsection{Dicke $^{3010}$}

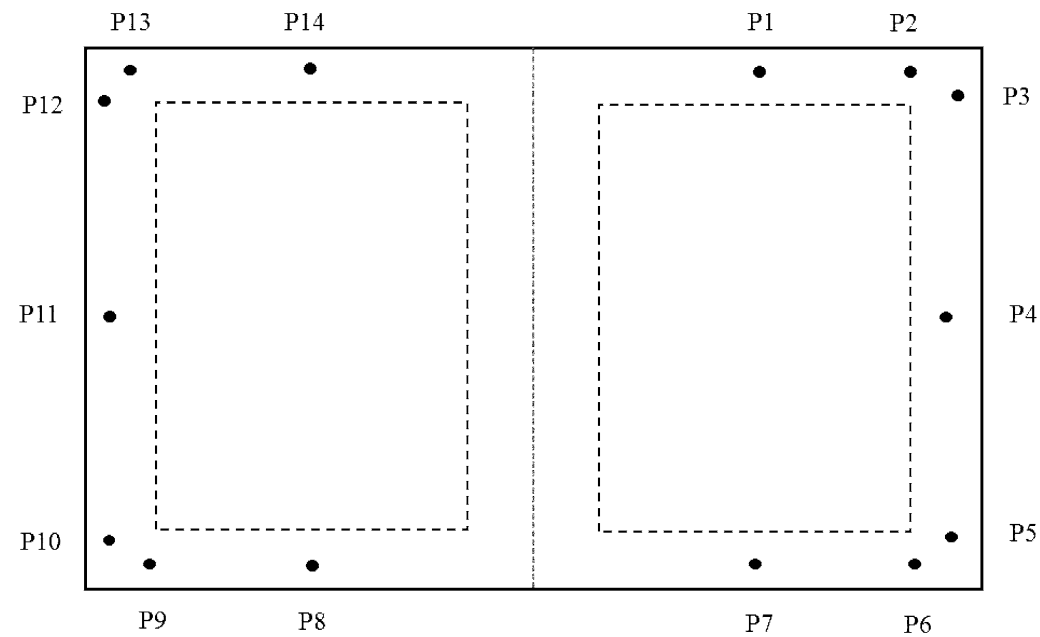

\begin{tabular}{|l|l|l|l|l|l|l|l|l|l|l|l|l|l|}
\hline P 1 & P 2 & P 3 & P 4 & P 5 & P 6 & P 7 & P 8 & P 9 & P 10 & P 11 & P 12 & P 13 & P 14 \\
\hline & & & & & & & & & & & & & \\
\hline
\end{tabular}

3010 Abgebildetes Schema nach Ornato et al. 2001, Bd. 1, 44. 


\begin{tabular}{lll}
\hline Kategorie & Messung & $\begin{array}{l}\text { Interpretationsmöglich- } \\
\text { keiten }\end{array}$ \\
\hline $\begin{array}{l}\text { Dicke in mm } \\
\text { größte Dickendifferenz } \\
\text { eines Bogens }\end{array}$ & & \\
$\begin{array}{l}(\mathrm{P} 1+\ldots+\mathrm{P} 14): 14= \\
\text { eine Ecke dicker }\end{array}$ & Welche & \\
$\square$ eine Seite dicker & Welche & $\begin{array}{l}\text { Verstärkung der Klaub- } \\
\text { spitze }\end{array}$ \\
& & $\begin{array}{l}\text { vom Schöpfgesellen } \\
\text { abgewandte Seite (franz. } \\
\text { bonne rive) }\end{array}$ \\
\end{tabular}

\subsection{Faserverteilung}

\begin{tabular}{lll}
\hline Phänomen & Interpretationsmöglichkeiten & Arbeitsschritt \\
\hline$\square$ wolkiges Papier & ungeübter Schöpfgeselle & Schöpfen \\
& wenig Entwässerungszeit & Schöpfen \\
& hohe Faserkonzentration in der Bütte & Schöpfen \\
& gefaulte Lumpen & Rohstoffaufbereitung: \\
& & Faulen \\
verteilung & geübter Schöpfgeselle & Schöpfen \\
& ausreichend Entwässerungszeit & Schöpfen \\
& ungefaulte Lumpen & Rohstoffaufbereitung: \\
\hline
\end{tabular}




\subsection{Siebstruktur ${ }^{3011}$}

Darstellung des Doppelblatts (Vorlage zum Ausfüllen, Beispiel siehe unten)

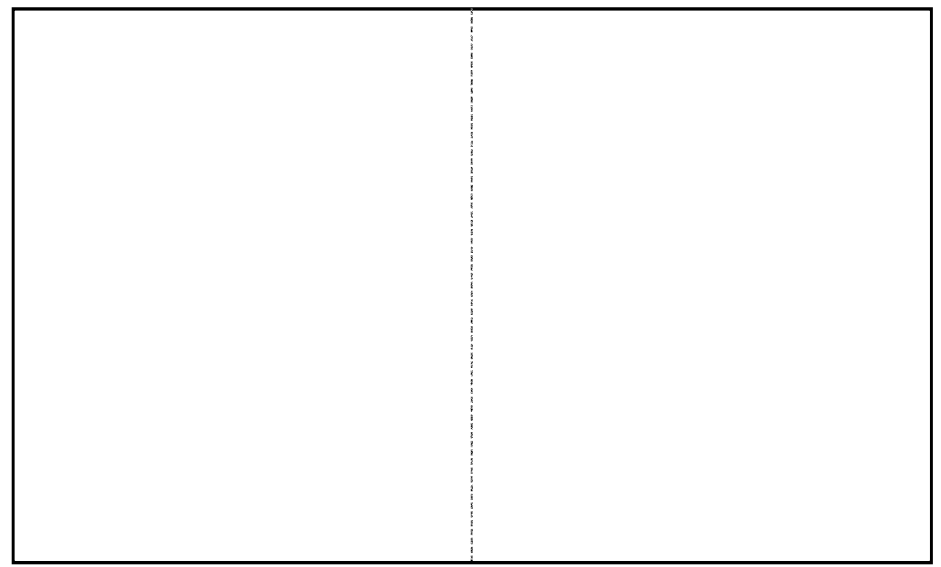

Beispiel einer Doppelblattdarstellung
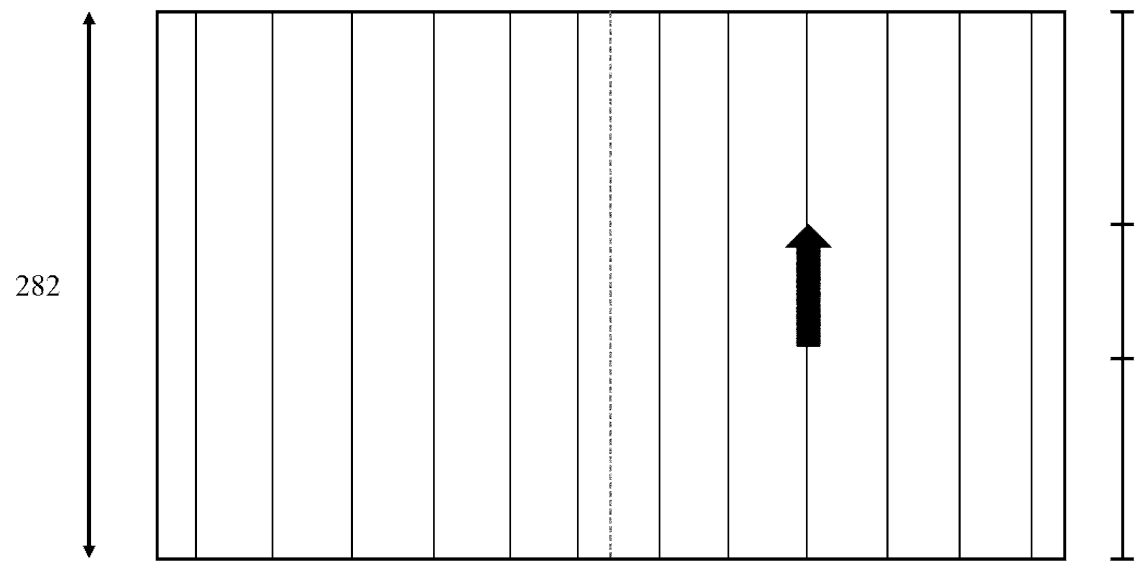

126

$17 \quad 38 \quad 38$

37
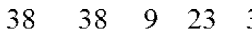

29

$\begin{array}{lll}38 & 37 & 18\end{array}$

422

3011 Schemata nach Bourlet/Bretthauer/Zerdoun Bat-Yehouda 2012/2017, 2f. 


\subsubsection{Ripplinien}

\begin{tabular}{|c|c|}
\hline Kategorie & Interpretationsmöglichkeiten \\
\hline $\begin{array}{l}\text { Anzahl auf } 10 \mathrm{~mm} \\
\text { ( } \emptyset \text { aus drei Messungen) }\end{array}$ & $\begin{array}{l}\text { weniger als } 6 \text { Ripplinien pro Zentimeter } \rightarrow \text { grobes } \\
\text { Papier mehr als } 8 \text { Ripplinien pro Zentimeter } \rightarrow \text { feines } \\
\text { Papier }\end{array}$ \\
\hline $\begin{array}{l}\text { Anzahl auf } 100 \mathrm{~mm} \\
\text { ( } \emptyset \text { aus drei Messungen) }\end{array}$ & \\
\hline $\begin{array}{l}\square \text { alternierende Folge von } \\
\text { dünnen und dicken Ripplinien }\end{array}$ & $\begin{array}{l}\rightarrow \text { grobe und feine Rippdrähte wurden zu einem Sieb verfloch- } \\
\text { ten, die tiefer liegenden, feineren Drähte ergeben nur schwache } \\
\text { Linien im Papier } \\
\rightarrow \text { Straffen nur eines Kettdrahts, der dann jeden zweiten Ripp- } \\
\text { draht nach unten drückt beziehungsweise nach oben hebt }\end{array}$ \\
\hline $\begin{array}{l}\square \text { in regelmäßigen Abständen } \\
\text { und parallel zu den Ripplinien } \\
\text { verlaufende dunkle Streifen, } \\
\text { sog. Kannelierung }\end{array}$ & $\begin{array}{l}\rightarrow \text { auf mehrere stärkere Rippdrähte folgt ein dünnerer } \\
\rightarrow \text { dunkle Streifen entstehen an den Stellen, an denen der } \\
\text { Kettdraht auf den Steg genäht wurde, dort liegt das Sieb minimal } \\
\text { enger an den Stegen an, sodass sich dort mehr Faserbrei } \\
\text { sammelt }\end{array}$ \\
\hline
\end{tabular}

\subsubsection{Kettlinien}

\begin{tabular}{ll}
\hline Kategorie & Analyse \\
\hline Anzahl Kettlinien & \\
$\varnothing$ Abstand zwischen den Kettlinien (in mm) & \\
Nähspuren auf den Kettlinien & $\square$ regelmäßig \\
& $\square$ vereinzelt \\
& $\square$ keine \\
Deformationen der Kettlinien & $\square$ nein \\
& $\square$ ja \\
Randlinie (tranchefile) & $\square$ nein \\
& $\square$ ja Abstand zum Rand: \\
\hline
\end{tabular}




\subsubsection{Stegschatten}

\begin{tabular}{ll}
\hline Stegschatten & Interpretationsmöglichkeiten \\
\hline$\square$ deckungsgleich mit Kettlinien & Stege unter den Kettdrähten \\
$\square$ mittig zwischen den Kettlinien & Stege mittig zwischen den Kettdrähten \\
$\square$ unregelmäßige Verteilung & Stege in unregelmäßigem Abstand \\
$\square$ ohne Schatten & Sieb mit Untergewebe (Papier nach 1800) \\
\hline
\end{tabular}

\subsection{Wasserzeichen ${ }^{3012}$}

Position des Wasserzeichens auf einem kompletten Bogen nach Benutzungsrichtung

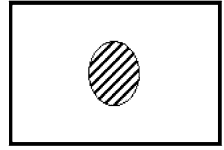

I

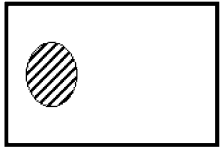

II

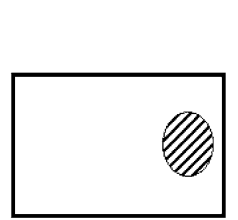

III

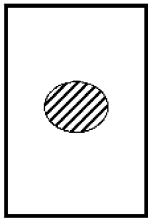

IV

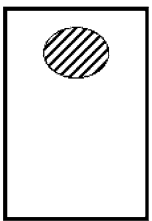

V

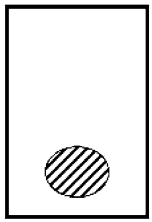

VI

\begin{tabular}{ll}
\hline$\square I$ & Bogen horizontal, Wasserzeichen mittig \\
\hline$\square I I$ & Bogen horizontal, Wasserzeichen auf der linken Bogenhälfte \\
\hline$\square I I I$ & Bogen horizontal, Wasserzeichen auf der rechten Bogenhälfte \\
\hline$\square I V$ & Bogen vertikal, Wasserzeichen mittig \\
\hline$\square V$ & Bogen vertikal, Wasserzeichen in der oberen Hälfte \\
\hline$\square \mathrm{VI}$ & Bogen vertikal, Wasserzeichen in der unteren Hälfte \\
\hline
\end{tabular}

3012 Schemata nach Bourlet/Bretthauer/Zerdoun Bat-Yehouda 2012/2017, 2. Für eine ausführliche Betrachtung des Wasserzeichens, die hier nicht Gegenstand sein soll, vgl. P. Tschudin 2012a, Anhang II, 282-284, 289-293. 
Position des Wasserzeichens in Bezug auf die Faltung



A

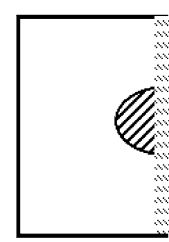

B

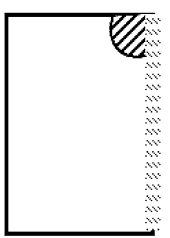

C

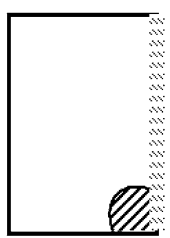

D

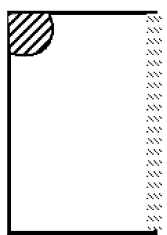

E

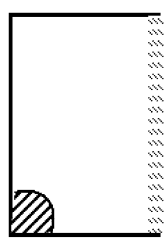

$F$

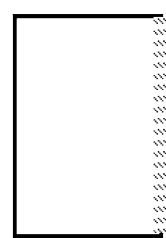

G

\begin{tabular}{ll}
\hline$\square \mathrm{A}$ & Folio-Format (Lage: 1 Bl. mit WZ, 1 Bl. ohne WZ) \\
\hline$\square \mathrm{B}$ & Quart-Format (Lage: 2 Bl. mit WZ, 2 Bl. ohne WZ) \\
\hline$\square \mathrm{C}$ & Oktav-Format (Lage: 4 Bl. mit WZ, 4 Bl. ohne WZ) \\
\hline$\square \mathrm{D}$ & Oktav-Format (Lage: 4 Bl. mit WZ, 4 Bl. ohne WZ) \\
\hline$\square E$ & Duodez-Format (Lage: 4 Bl. mit WZ, 8 Bl. ohne WZ) \\
\hline$\square \mathrm{F}$ & Duodez-Format (Lage: 4 Bl. mit WZ, 8 Bl. ohne WZ) \\
\hline$\square \mathrm{G}$ & Folio-Format; Quart-Format; Oktav-Format; Duodez-Format \\
\hline
\end{tabular}

Position des Wasserzeichens in Bezug zu den Kettlinien

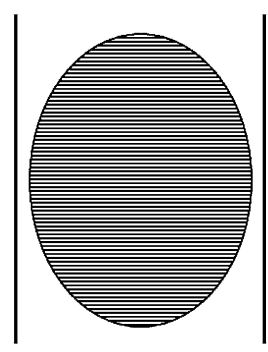

A

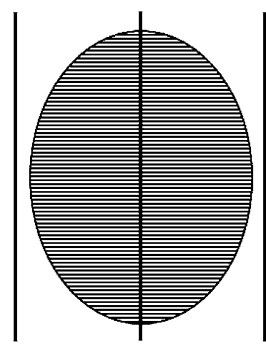

B

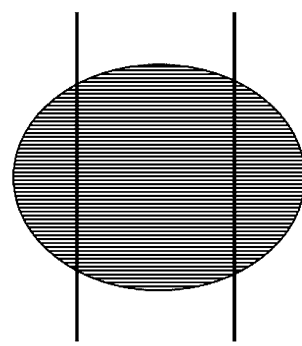

C

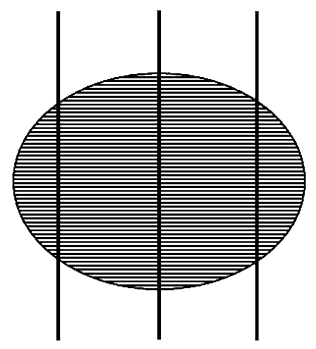

D

\begin{tabular}{ll}
\hline$\square A$ & Wasserzeichen zwischen zwei Kettlinien \\
\hline$\square B$ & Wasserzeichen zwischen zwei Kettlinien mit Hilfslinie \\
\hline$\square C$ & Wasserzeichen über zwei Kettlinien \\
\hline$\square D$ & Wasserzeichen über zwei Kettlinien mit Hilfslinie \\
\hline Nähspuren & $\begin{array}{l}\square \text { ja } \\
\square \text { nein }\end{array}$ \\
\hline
\end{tabular}




\subsection{Fehler im Papier}

\begin{tabular}{|c|c|c|}
\hline Phänomen & Interpretationsmöglichkeiten & Arbeitsschritt \\
\hline \multirow[t]{4}{*}{$\begin{array}{l}\square \text { Knoten und Unregel- } \\
\text { mäßigkeiten im Papier }\end{array}$} & $\begin{array}{l}\text { unsorgfältiges Sortieren: Mischen von sehr } \\
\text { abgetragenen und fast neuen sowie von feinen } \\
\text { und groben Stoffen }\end{array}$ & $\begin{array}{l}\text { Rohstoffaufbereitung: } \\
\text { Sortieren der Lumpen }\end{array}$ \\
\hline & kurze, unzureichende Stampfdauer & $\begin{array}{l}\text { Rohstoffaufbereitung: } \\
\text { Stampfen }\end{array}$ \\
\hline & $\begin{array}{l}\text { getrocknete Faserreste vom Büttenrand oder } \\
\text { dem Rand des Zeugkastens }\end{array}$ & Ganzzeugaufbewahrung \\
\hline & schlecht gerührter Faserbrei in der Bütte & Schöpfen \\
\hline \multirow{4}{*}{$\begin{array}{l}\square \text { Falten und Überlap- } \\
\text { pungen }\end{array}$} & unsauberes Abrollen des Siebs auf dem Filz & Gautschen \\
\hline & schnelles Arbeiten unter Zeitdruck & Gautschen \\
\hline & unerfahrener Gautscher & Gautschen \\
\hline & unvorsichtiger oder unerfahrener Leger & Legen \\
\hline \multirow[t]{3}{*}{$\square$ Überdehnung } & unsauberes Abrollen des Siebs auf dem Filz & Gautschen \\
\hline & schnelles Arbeiten unter Zeitdruck & Gautschen \\
\hline & unerfahrener Gautscher & Gautschen \\
\hline \multirow[t]{3}{*}{$\square$ ausgerissene Ecken } & $\begin{array}{l}\text { Bogen haftet stark am Filz, bei Überdehnun- } \\
\text { gen reißt die schwächste Stelle }\end{array}$ & $\begin{array}{l}\text { Rohstoffaufbereitung: } \\
\text { Faulen }\end{array}$ \\
\hline & unvorsichtiger Leger & Legen \\
\hline & $\begin{array}{l}\text { schlechtes Entwässern von Pulpe aus gefaul- } \\
\text { ten Lumpen }\end{array}$ & $\begin{array}{l}\text { Rohstoffaufbereitung: } \\
\text { Faulen }\end{array}$ \\
\hline \multirow[t]{2}{*}{$\square$ Fingerabdrücke } & $\begin{array}{l}\text { Leger hat den Bogen mit den Finger- } \\
\text { spitzen hochgehoben }\end{array}$ & Legen \\
\hline & unerfahrener Leger & Legen \\
\hline$\square$ Fremdkörper & $\begin{array}{l}\square \text { Haare } \\
\square \text { Holzsplitter } \rightarrow \text { von der Bütte, dem } \\
\text { Rührgerät oder der Schöpfform } \\
\text { Häufiges Vorkommen spricht für eine nachläs- } \\
\text { sige Instandhaltung der Arbeitsgeräte } \\
\square \text { Sonstiges }\end{array}$ & Schöpfen \\
\hline$\square$ rot-braune Flecken & $\begin{array}{l}\text { Rost vom Eisen der Stampfhämmer oder des } \\
\text { Stampftrogs }\end{array}$ & $\begin{array}{l}\text { Rohstoffaufbereitung: } \\
\text { Stampfen }\end{array}$ \\
\hline
\end{tabular}




\begin{tabular}{lll}
\hline Phänomen & Interpretationsmöglichkeiten & Arbeitsschritt \\
\hline $\begin{array}{l}\text { kreisrunde helle } \\
\text { Stellen }\end{array}$ & Wassertropfen & $\begin{array}{l}\text { Schöpfen } \\
\text { Gautschen }\end{array}$ \\
$\begin{array}{l}\text { runde bis ovale helle } \\
\text { Stellen mit dunklem }\end{array}$ & $\begin{array}{l}\text { Brillen (beim Gautschen entstehende Spuren } \\
\text { von Luftblasen) } \\
\text { Rand }\end{array}$ & $\begin{array}{l}\text { Gautschen } \\
\text { einer der ersten Bogen eines Pauschts }\end{array}$ \\
$\begin{array}{l}\square \text { Vorkommen von } \\
\text { Fehlern }\end{array}$ & $\begin{array}{l} \\
\text { möglicherweise unter Zeitdruck }\end{array}$ \\
& $\square$ moderat \\
& $\square$ selten $\rightarrow$ sorgfältiges Arbeiten & \\
\hline
\end{tabular}




\subsection{Anhang V}

Gläubiger des Basler Papiermachers Hans Züricher im Konkursverfahren von $1494^{3013}$ 



\begin{tabular}{|c|c|c|c|}
\hline & Datum & Person & Forderung \\
\hline 1. & 30.04 .1494 & Steffann Hanns Zürichers knecht & 15 Gulden 3 Schilling \\
\hline 2. & 30.04 .1494 & Jacob von Rinach & 7 Pfund 5 Schilling \\
\hline 3. & 30.04 .1494 & Adam sin leger & 36 Schilling \\
\hline 4. & 30.04 .1494 & Hanns von Schaffhusenn der bappirmacher & 13 Pfund minus 3 Schilling \\
\hline 5. & 30.04 .1494 & Hanns Nussboum & 9 Pfund 5 Schilling \\
\hline 6. & 30.04 .1494 & Ennelin lumpenzerrerin & 6 Pfund 15 Schilling \\
\hline 7. & 30.04 .1494 & Frölicher der Metzger & 7 Pfund \\
\hline 8. & 02.05 .1494 & Herr Hanns Wesslin & 21 Pfund 17 Schilling 1 Pfennig \\
\hline 9. & 02.05 .1494 & Claws von Rasstatt der küffer & 5,5 Pfund 2 Schilling 2 Pfennig \\
\hline 10. & 02.05 .1494 & Hanns Ber & 2 Pfund \\
\hline 11. & 04.05 .1494 & Agnes Zschany die lumpenzerrerin & 4 Pfund \\
\hline 12. & 06.05 .1494 & Peter von Büsch & 8 Pfund \\
\hline 13. & 09.05 .1494 & Adam Lamp & 4,5 Pfund \\
\hline 14. & 09.05 .1494 & Galus Zinck von Medet & 2 Pfund 5 Schilling \\
\hline 15. & 10.05 .1494 & Fridlin Beltz der snider & für 6,5 Pfund \\
\hline 16. & 10.05 .1494 & $\begin{array}{l}\text { Hanns von Bobenhüsen/Hanns Vischers } \\
\text { erben }\end{array}$ & 2 Pfund \\
\hline 17. & 10.05 .1494 & Meister Michel der bappirer & 2 Gulden \\
\hline 18. & 10.05 .1494 & Meister Hanns Ammerbach der trugker & 5 Gulden \\
\hline 19. & 10.05 .1494 & Lienhart Hug von Bromberg & 2 Pfund 5 Schilling \\
\hline 20. & 10.05 .1494 & Claws Brottbegk von Mutetz & 19 Schilling \\
\hline 21. & 12.05 .1494 & Elsin Zürichers junckfrow & 18 Schilling \\
\hline 22. & 13.05 .1494 & Ulrich Ysenflam & 16 Schilling \\
\hline 23. & 13.05 .1494 & $\begin{array}{l}\text { Herr Michel Schaffner Convent herr zu } \\
\text { Sannt Albann }\end{array}$ & 2 Pfund \\
\hline 24. & 15.05 .1494 & Caspar Helg des Zürichers knecht & 1 Pfund 4 Schilling \\
\hline 25. & 15.05 .1494 & Claus von Anndernach & 3 Pfund 18 Pfennig \\
\hline 26. & 15.05 .1494 & Gerichtschriber & 9 Schilling \\
\hline 27. & 15.05 .1494 & Meister Lienhart Davidt & 18 Schilling \\
\hline 28. & 15.05 .1494 & Clewin Ritter von Otligken & 35 Schilling \\
\hline 29. & 28.05 .1494 & Conratt Weßlin & Pfändung zweier Papiermühlen \\
\hline 30. & 03.06 .1494 & Jacob Warne der küffer & 14 Schilling \\
\hline
\end{tabular}


